\title{
ADAPTIVE CONTROL OF SINGLE-INPUT SINGLE-OUTPUT HYBRID SYSTEMS POSSESSING INTERACTING DISCRETE- AND CONTINUOUS-TIME DYNAMICS
}

\author{
M. DE LA SEN
}

Received 26 January 2005

This paper deals with the problem of synthesizing a robust adaptive controller for a specific class of single-input single-output (SISO) time-invariant hybrid controlled object (plant) which can operate under bounded disturbances and/or unmodeled dynamics. The hybrid plant dealt with is composed of two coupled subsystems, one of them being of continuous-time type while the other is digital. As a result there are also mixed continuous-time and discrete signals present in the system associated either with the solutions of differential equations which depend at the same time on both discrete-time and continuous-time forcing terms and on generalized difference equations associated with discretized and digital signals. The estimation algorithm is of a continuous-time nature since the plant parameter estimates are updated for all time. It also incorporates a relative adaptation dead-zone as a robust stabilization mechanism which prevents against instability in the presence of a common class of unmodeled dynamics and bounded noise.

\section{Introduction}

A very important class of hybrid systems are those being interacting networks of digital and continuous systems which have, in general, coupled dynamics. They commonly arise in interactive distributed simulation, plant process and traffic control as well as robot design and path planning. This class of hybrid systems has received an important attention in the last years since its formulation is very close to the modeling requirements of real processes consisting of coupled combinations of continuous-time and digital and/or discrete-time subsystems, $[3,6,7,8,16,29]$. The study of the properties as well as the synthesis of the hybrid models has led to an emerging design tool in theoretical and practical control applications. Such systems are important mainly because of the frequent use of digital computers for active control of continuous-time systems and because many dynamic systems possess digital integrated components in usual applications in the Telecommunications field. They are also appropriate for modeling many existing Biological Processes in the Nature which evolve in a continuous manner with time while being also driven by discrete events which modify their parameterizations or states. Additional 
reasons which emphasize the importance of such systems areas described in studies in $[7,29]$ concerned with:

(i) The classical tool for dealing with inter-sampling performance with the use of the modified $z$-transform implicitly requires discretization of the inputs.

(ii) Sampled-data systems may have innocuous sample-time dynamics combined with unacceptable sampling ripples.

Such models describe appropriately dynamic systems which consist of coupled continuous-time and digital sub-states while being, in general, subject to simultaneous continuous-time and discrete-time control actions. (see [3, 6, 7, 8, 16, 29]). An important subclass of such systems includes those involving the use of discrete-time controllers for continuous-time plants. These configurations are of particular interest in adaptive control. Another type of hybrid adaptive controllers involves discrete-time updating of their parameters by continuous-time regressors while the plant control action generated is of a continuous-time nature (see, for instance, $[3,6,16,29]$ ). Recently, the synthesis of robust adaptive stabilizers for the above mentioned class of hybrid systems involving a first-order continuous sub-state has been addressed in [8] by using covariance resetting.

On the other hand, recent research in adaptive control has been focused on obtaining robustness results for the case when the system operates under bounded noise and unmodeled dynamics. The research is being specifically addressed towards the relaxation of classical assumptions on the plant and its working operation modes. The technique used in [8], and references therein, to avoid singularities has been the estimates modification so that the modified estimated plant model remains controllable for all time and at the limit. This paper addresses the problem of robust adaptive control of the class of non necessarily inversely stable single-input single-output linear hybrid plants which are composed of coupled linear continuous and digital subsystems. Three major design guidelines are involved in the controller synthesis, namely:

(1) The synthesized controller is of pole-placement type (see, for instance, $[4,9,11,14$, $21,33,38])$ and it is inspired in the so-called internal model control principle $([4,11])$ rather than in a tracker-type design $[5,12]$.

(2) The parameter updating algorithm is implemented with a relative dead zone which is built according to the relative size of the contributions of the uncertainties to the output compared to those of the prediction error. The scheme freezes the parameter adaptation process when the prediction error is small compared to the size of the contribution of the uncertainties to the closed-loop action (see $[8,9,14])$.

(3) The estimation scheme is of a continuous-time nature in the sense that all the plant parameters, even those arising from couplings from the discrete description at sampling instants, are estimated at all time. This leads to an associate problem of stability of nonlinear dynamic systems subjected to mixed continuous-time/discrete-time time-varying parameterizations, since the estimation is inherently time-varying by nature, $[13,23,24,40]$ rather than to a simpler problem of numerical stability, [23] where time is not usually a relevant argument for stability purposes.

The hybrid nature of the system arises from the feature that the plant is simultaneously driven by the continuous time input plus its samples at sampling instants. This intuitively means that with only a generated input signal, two effective signals are involved 
to drive the system, the second one being its samples at sampling points operated by a zero-order-hold. As a result, its input-output differential equation has forcing terms generated by the system description at sampling instants. All the relevant signals in the system are filtered through stable filters to improve the adaptation transients and robustness against noise and unmodeled dynamics. The estimation scheme involves the estimation of both the continuous-time and discrete-time parameters in a continuous fashion what emphasizes the hybrid nature of the system. Those issues have to be taken into account in the stability analysis where the time-differential operator or, equivalently, (in a formal sense) the Laplace operator and the discrete-delay operator or, equivalently, (in a formal sense) the $z$-transform operator are involved.

In a general context of hybrid systems, the class of object considered here is the adaptive control of a plant (or controlled object) which is linear and hybrid in the sense that it contains coupled continuous-time and digital subsystems. Many real situations concerning dynamic systems are included in this situation like, for instance, the control of continuous-time system through a discrete controller or the case of large-scale systems in which many components have continuous-time dynamics while others only evolve at sampling points. Furthermore, the problem has additional difficulties to be overcome, like, for instance the whole system to be stabilized requires recursive estimation (since its parameter are unknown) and the stability discussion requires nonlinear techniques of analysis since the whole problem becomes nonlinear because of the estimation process. Furthermore, both the estimation and stability analysis as well as the controller synthesis problem require the use of mixed continuous-time/discrete-time techniques because of the hybrid nature of the whole scheme with the additional drawback of the presence of mutual couplings. Also, the control function is usually discontinuous at the sampling points of the discrete subsystem. Hybrid systems sometimes incorporates also logic switching rules, $[15,34]$ or the use of numerical tools, [28]. They offer a wide range of models for applications in the fields of robotics, compartmental dynamic models, control of DC or induction servomotors, Fuzzy systems, neural networks or computational techniques (see, for instance, $[2,18,19,22,25,26,27,30,36,37]$ ). In particular, the use of so-called hybrid functions (block-pulse functions plus Legendre polynomials) is of usefulness in the analysis and optimal control of linear delay systems, [27]. On the other hand, the dissipativity theory of left-continuous dynamical systems includes many other particular results for dynamic systems, [18].

The paper is organized as follows. Section 2 establishes the state-space structure of the nominal hybrid plant and discusses its filtered input/output descriptions obtained from a state-space description. An extension of such a model to the case of presence of unmodeled dynamics and bounded disturbances is obtained by including an additive disturbance function to describe the above uncertainties. Section 3 is devoted to the robust adaptive control scheme and associated parameter estimation algorithm. Relative dead zones are used to prevent the adaptation process against closed-loop instability caused by prediction errors being small compared to the size of the contributions of the uncertainties to the output. Related techniques were used in [8] for a class of first-order hybrid systems and in [9] for time-delay systems. Section 4 is concerned with the convergence properties of the estimates as well as to the closed-loop robust stability. Section 5 presents 
some issues concerning the identification of the nominal uncontrolled hybrid plant and, finally, conclusions end the paper.

\section{Hybrid plant and problem statement}

2.1. Nominal plant. Consider the linear and time-invariant SISO hybrid system

$$
\begin{gathered}
\dot{x}_{c}(t)=A_{c} x_{c}(t)+A_{d c} x_{d}[k]+b_{c} u(t)+b_{d c} u[k], \\
x_{d}[k+1]=A_{d} x_{d}[k]+A_{c d} x_{c}[k]+b_{d} u[k], \\
y(t)=c_{c}^{T} x_{c}(t)+c_{d}^{T} x_{d}[k]+d_{c} u(t)+d_{d} u[k]
\end{gathered}
$$

for all $t \in[k T,(k+1) T)$ and all integer $k \geq 0$ where $x_{c}(t)$ and $x_{d}[k]$ are, respectively, the continuous-time and digital sub-states of respective dimensions $n_{c}$ and $n_{d}, u(t)$ and $y(t)$ are the scalar continuous-time input and output and $T$ is the sampling period. Subscripts " $c$ " and " $d$ " in the above formulas are used for denoting the parameters of the continuous and digital subsystems or the gains or matrix gains from a continuous-time or discretetime/digital signal, respectively. On the other hand, subscripts " $c d$ " and " $d c$ " are used for couplings from the digital system to the continuous one and vice-versa, respectively, in order to easily relate notation with intuition. In particular, $d_{c}$ and $d_{d}$ are the scalar interconnection input-output gains associated with the continuous-time input $u(t)$ and its sampled values $u(k T)=u[k]$ so that the zero state-output is $y(t)=d_{c} u(t)$ if $t \neq k T$ and $y[k]=y(k T)=d_{c} u(k T)+d_{d} u[k]=\left(d_{c}+d_{d}\right) u[k]$. The notation $z[k]$ applies to either a digital signal or to a sampled continuous one at time $t=k T$. All the matrices in (2.1) are of dimensions being compatible with the corresponding vectors. Note that the system (2.1) is driven simultaneously by $u(t)$ and $u[k]=u(k T)$ for all $t \in[k T,(k+1) T)$. The controllability and observability properties of (2.1) have been studied in [7].

In the following, $\operatorname{Det}(\cdot), \operatorname{Tr}(\cdot), \operatorname{Adj}(\cdot), \lambda_{\max }(\cdot)$, and $\lambda_{\min }(\cdot)$ denote, respectively, the determinant, trace, adjoint matrix and maximum and minimum eigenvalue of the $(\cdot)$ matrix and $I$ is the identity matrix of appropriate order depending on the context. The $l_{2}-$ norms of vector and matrices are denoted by $\|\cdot\|_{2}$. Also, $D^{i}=d^{i} / d t^{i}, s, q$, and $z$ stand for the $i$ th order time-derivative, Laplace, time advance and $z$-transform operators, respectively. Note that $D$ is formally equivalent to $s$ and $q$ is formally equivalent to $z$. The various signals are denoted similarly, except in the argument, if they are expressed in the time and transform domains (i.e., $s$ replaces $D$ and $z$ replaces $q$ ). $L_{p} \equiv L_{p}(0, \infty)$ and $L_{\infty} \equiv L_{\infty}(0, \infty)$ denote, respectively, the spaces of $p$-Lebesgue integrable ( $p$ being any positive real number) and uniformly essentially bounded real functions of time on $[0, \infty)$ endowed with the respective norms:

$$
\begin{gathered}
\|f\|_{p}=\left(\int_{0}^{\infty}\|f(\tau)\|^{p} d \tau\right)^{1 / p}, \quad \forall f \in L_{p}, \\
\|f\|_{\infty}=\operatorname{Inf}\left(C \geq 0: \operatorname{esssup} \operatorname{Ind}_{t \geq 0}\|f(t)\| \leq C\right), \quad \forall f \in L_{\infty} .
\end{gathered}
$$

If $f$ is a real vector function $f$ is replaced with $\left(f^{T} f\right)^{1 / 2}$ but we keep the notations $f \in L_{p}$, $f \in L_{\infty}$ without specifying the dimension of $f$ in $L_{p}$ and $L$ in order to keep a simple 
notation. No confusion is expected since the dimensions are clear from the context in all the paper body. For subsequent use, define filtered signals:

$$
\begin{gathered}
y_{f}(t)=\left(\frac{1}{F(D)}\right) y(t) ; \quad u_{f}(t)=\left(\frac{1}{F(D)}\right) u(t), \\
\omega_{f}(t)=\left(\frac{1}{F(D)}\right) \omega[k] ; \quad z_{f}(t)=\left(\frac{1}{F(D)}\right) z[k] \quad \text { for } t \in[k T,(k+1) T), \\
\omega[k]=A_{d c} x_{d}[k]+b_{d c} u[k] ; \quad z[k]=c_{d}^{T} x_{d}[k]+d_{d} u[k]
\end{gathered}
$$

under initial conditions $D^{i} y_{f}(0), D^{i} u_{f}(0), D^{i} \omega_{f}(0)$, and $D^{i} z_{f}(0)$ for $i=0,1, \ldots, n_{c}-1$, where $F(D)=D^{n_{c}}+\sum_{i=1}^{n_{c}} f_{i} D^{n_{c}-i}$ is a strictly Hurwitzian monic polynomial of degree $n_{c}$. The signal $\omega[k]$ defined in (2.3b) and (2.3c) is a coupling signal from the discrete subsystem to the discretized continuous one of state $x_{c}[k]=x_{c}(k T)$ through the combined solution of (2.1). The subsequent result is concerned with the input-output description of (2.1) and it is proved in Appendix A. Note that the hybrid system (2.1) is simultaneously driven by the current input and its sampled value at the preceding sampling instant. If the input is discontinuous, but bounded, at sampling instants then the control effect on the continuous-time sub-state is similar as having two independent input channels, $u(t)$ and $u[k]=u(k T)$ at each current time $t$. That property follows since isolated bounded discontinuities $u[k]=u(k T)$ of $u(t)$ do not modify their contribution to $x_{c}(t)$ while $u[k]$ drives independently $x_{d}[k]$. Note that there are two kinds of relevant information in the same input signal through the above description, namely, a digital signal $u[k]$, which is of low frequency for large sampling periods, and a continuous-time one $u(t)$ with possible discontinuities at sampling instants, which can be of high frequency if it is fast varying or of low frequency, otherwise. This occurs since input discontinuities at the sampling points of the digital subsystem are admitted; that is, $u\left(k T^{-}\right) \neq u\left(k T^{+}\right)=u[k]$. Then, the discontinuities at sampling points $u\left(k T^{+}\right)=u[k]$ may be designed independently of the inter-sample values of $u(t) t \in(k T,(k+1) T)$, for instance, by using simultaneously a continuous-time controller and digital ones. In this context, $u(t)$ and $u[k]$ act as two distinct input channels in the hybrid system as (2.1) emphasize. Such a design philosophy can be of potential applicability in transmission of modulated signals through a carrier. Note that the isolated bounded discontinuities of $u(t)$ at sampling points do not influence the evolution equation of the continuous subsystem through the continuous in. Note also that the appearance of a direct, possibly nonzero, transmission $d_{d}$ in (2.1c) is just made for generalization purposes in order to admit within the class of given systems those having discrete transfer functions of zero relative degree after the discretization is performed.

Leмma 2.1. The differential filtered input-output description is given by

$$
\begin{aligned}
\alpha(D) y_{f}(t)= & \beta(D) u_{f}(t)+\sum_{i=0}^{n}\left(\beta_{1 i}(D) y_{f}[k-i]+\beta_{2 i}(D) u_{f}[k-i]\right) \\
& +\sum_{i=1}^{n} \int_{0}^{T} \beta_{3 i}(D, \tau) u_{f}[(k-i) T+\tau] d \tau+v_{i c}(t)
\end{aligned}
$$


for $t \in[k T,(k+1) T)$ for all integer $k \geq 0$ with $n=n_{c}+n_{d}$, and the various polynomials parameterizing the description are directly obtained from the determinant and adjoint of the polynomial matrix $\left(D I-A_{c}\right)$ as follows:

$$
\begin{gathered}
\alpha(D)=\operatorname{Det}\left(D I-A_{c}\right)=\sum_{i=0}^{n_{c}} \alpha_{i} D^{n_{c}-i} ; \\
\beta(D)=\beta_{1}^{T}(D) b_{c}+d_{c} \alpha(D)=\sum_{i=0}^{n_{c}} \beta_{i} D^{n_{c}-i}, \\
\beta_{1}^{T}(D)=c_{c}^{T} \operatorname{Adj}\left(D I-A_{c}\right)=\sum_{k=1}^{n_{c}} \beta_{1 i}^{T} D^{n_{c}-k} ; \quad \beta_{i j}(D)=\sum_{k=0}^{n_{c}} \beta_{k}^{i j} D^{n_{c}-k} \quad(i=1,2 ; j=0,1, \ldots, n), \\
\beta_{3 j}(D, \tau)=\sum_{k=0}^{n_{c}} \beta_{k}^{3 j}(\tau) D^{n_{c}-k} \quad(j=1,2, \ldots, n), \\
v_{i c}(t)=c_{F}^{T} e^{A_{F} t} x^{*}(0)=c_{F}^{T} e^{A_{F} t}\left(\alpha(D)\left(x_{y}(0)-x_{z}(0)\right)-\beta(D) x_{u}(0)-\beta_{1}^{T}(D) x_{\omega}(0)\right)
\end{gathered}
$$

is the response to initial conditions of the continuous-time filters (2.3a) and (2.3b) with respective initial states $x_{y}(0), x_{u}(0), x_{z}(0)$, and $x_{\omega}(0)$ associated with some respective statespace realizations $R_{F}=\left(c_{F}^{T}, A_{F}, b_{F}\right)$ of the various identical filters $1 / F(s)$ used in (2.3a) and (2.3b) to generate as outputs the filtered output, input and remaining signals from their corresponding unfiltered versions.

Remark 2.2. All time-derivatives of signals at sampling instants are taken to the right. Note from (2.7) that $\operatorname{deg}(\beta) \leq \operatorname{deg}(\alpha)=n_{c}$ in (2.11b) with the inequality being strict if and only if $d_{c}=0$. Note that there is one parameter more than the minimum required to specify (2.4a) in the sense that $\alpha_{0}$ can be fixed to unity (i.e., $\alpha(D)$ can be chosen monic) by using normalization if necessary in those equations. Note that at the sampling instant $t=k T$, the description (2.4a) becomes

$$
\begin{aligned}
\left(\alpha(D)-\beta_{10}(D)\right) y_{f}[k]= & \left(\beta(D)+\beta_{20}(D)\right) u_{f}[k]+\sum_{i=1}^{n}\left(\beta_{1 i}(D) y_{f}[k-i]+\beta_{2 i}(D) u_{f}[k-i]\right) \\
& +\sum_{i=1}^{n} \int_{0}^{T} \beta_{3 i}(D, \tau) u_{f}[(k-i) T+\tau] d \tau+v_{i c}(t)
\end{aligned}
$$

after grouping corresponding coefficient polynomials operating on $y_{f}[k]$ and $u_{f}[k]$ in (2.4a). Such a description makes obvious that, in general, the plant parameterization of the differential input-output model of the nominal hybrid plant is, in general, different "in-between" and "at" sampling instants.

Remark 2.3. Since $\alpha(D)=D^{n_{c}}+\sum_{i=1}^{n_{c}} \alpha_{i} D^{n_{c}-i}, \alpha(D) x_{y}(0)=D^{n_{c}} x_{y}(0)+\sum_{i=1}^{n_{c}} \alpha_{i} D^{n_{c}-i} x_{y}(0)$ where the values of $D^{j} x_{y}(0)$ for $j=1,2, \ldots, n_{c}$ are obtained from $D^{j+1} x_{y}(0)=A_{F} D^{j} x_{y}(0)$ $+b_{F} D^{j} u(0)$ which follow by recursive calculations from $j=0$ to $2 n_{c}$ which corresponds 
to the state-space realization $R_{F}$ of dimension $2 n_{c}+1$ of the output filtering operation. It remains now to calculate $D^{(\cdot)} u(0)$ to be used in the above identities. Direct calculations from the input filtering equations yields $D^{j} u(0)=\left(1 / c_{F}^{T} b_{F}\right)\left(D^{j+1} u_{f}(0)-c_{F}^{T} A_{F} D^{j} x_{u}(0)\right)$ for $j=0,1, \ldots, 2 n_{c}$ and $D^{2 n_{c}+1} u_{f}(0)=D^{2 n_{c}+1} u(0)-\sum_{j=1}^{2 n_{c}+1} f_{j} D^{2 n_{c}+1-j} u_{f}(0)$. Similar calculations can be related to the initial conditions of the remaining continuous-time filter equations so that $x^{*}(0)$ is calculated in (2.8).

2.2. Non-ideal plant. Assume now that bounded noise and unmodeled dynamics are present in (2.1). In this case, the input-output description (2.4a) becomes

$$
\begin{aligned}
\alpha(D) y_{f}(t)= & \beta(D) u_{f}(t)+\sum_{i=0}^{n}\left(\beta_{1 i}(D) y_{f}[k-i]+\beta_{2 i}(D) u_{f}[k-i]\right) \\
& +\sum_{i=1}^{n} \int_{0}^{T} \beta_{3 i}(D, \tau) u_{f}((k-i) T+\tau) d \tau+\bar{v}_{i c}(t)+\eta_{f}(t)
\end{aligned}
$$

for $t \in[k T,(k+1) T)$ where $\bar{v}_{i c}(t)=v_{i c}(t)+\tilde{v}_{i c}(t)=c_{F}^{T} e^{A_{F} t} \bar{x}^{*}(0)=c_{F}^{T} e^{A_{F} t}\left(x^{*}(0)+\tilde{x}^{*}(0)\right)$, with $\tilde{v}_{i c}(t)=c_{F}^{T} e^{A_{F} t} \tilde{x}^{*}(0)$ are exponentially vanishing signals due to the contribution of the initial conditions of the uncertain dynamics defined by constant vectors in the continuous-time filtered equations and whose structure is similar to (2.8). Since those signals are exponentially vanishing the values of the involved constant vectors are not relevant to the adaptive controller synthesis. The input-output model (2.10) can be specialized at sampling instants from the nominal one given in Remark 2.2. The signal $\eta_{f}(t)$ is the filtered contribution of the unmodeled dynamics. Assume with no loss in generality that $\alpha(D)$ in Lemma 2.1 is monic (see Remark 2.2). The couplings caused by sampled signals from the digital to the continuous-time description make the continuous-time input to influence the continuous-time sub-state at sampling instants through (2.1a). This fact follows from the structure of (2.1) and it causes couplings into the digital sub-state trough (2.1b) and, then, input and state discontinuities at sampling instants in the time-differential input-output description (2.10). Such a phenomenon becomes explicit in the right-hand side of the input-output model (2.10) subject to noise and unmodeled dynamics but also in the perfectly modeled situation (i.e., $\eta_{f} \equiv 0$ so that no noise/unmodeled dynamics is present), dealt with in Lemma 2.1 since it is due to the hybrid character of the plant (2.1). This feature will cause a modification of the pole-placement control law of Section 4 related to the standard types used in purely continuous-time or discrete-time cases. The modification will consist in an extra additive signal in the control law at sampling instants. Another modification which will be used is the use of integral-type parameterizations which manipulate a dummy-time argument within the integral symbol apart from the continuous-time argument. Such a manipulation allows the definition of a standard-type Lyapunov's function candidate for the whole set of parameter estimates associated with both the continuous-time and digital subsystems.

The input-output model (2.10) can be expressed in regression form which are more appropriate for the subsequent presentation of the adaptive schemes. Note that (2.10) is 
identical to

$$
\begin{aligned}
y(t)= & (F(D)-\alpha(D)) y_{f}(t)+\beta(D) u_{f}(t)+\sum_{i=0}^{n}\left(\beta_{1 i}(D) y_{f}[k-i]+\beta_{2 i}(D) u_{f}[k-i]\right) \\
& +\sum_{i=1}^{n} \int_{0}^{T} \beta_{3 i}(D, \tau) u_{f}[(k-i) T+\tau] d \tau+\bar{v}_{i c}(t)+\eta_{f}(t) \\
= & \phi_{0}^{T}(t) \theta_{0}+\phi_{1}^{T}[k] \theta_{1}+\int_{0}^{T} \phi_{2}^{T}(k, \tau) \theta_{2}(\tau) d \tau+c_{F}^{T} e^{A_{F} t} \bar{x}^{*}(0)+\eta_{f}(t) \\
= & \int_{0}^{T} \phi^{T}(t, \tau) \theta(\tau) d \tau+\eta_{f}(t)
\end{aligned}
$$

for all $t \in[k T,(k+1) T)$ after using $(2.4 \mathrm{a})$ where

$$
\phi^{T}(t, \tau)=\left(\phi_{0}^{T}(t), \phi_{1}^{T}[k], \phi_{2}^{T}(k, \tau), c_{F}^{T} e^{A_{F} t}\right) ; \quad \theta^{T}(\tau)=\left(\frac{\theta_{0}^{T}}{T}, \frac{\theta_{1}^{T}}{T}, \theta_{2}^{T}(\tau), \frac{\bar{x}^{* T}(0)}{T}\right)
$$

with

$$
\begin{aligned}
\phi_{0}^{T}(t)= & \left(D^{n_{c}-1} y_{f}(t), \ldots, D y_{f}(t), y_{f}(t), D^{n_{c}} u_{f}(t), \ldots, D u_{f}(t), u_{f}(t)\right), \\
\phi_{1}^{T}[k]= & {\left[D^{n_{c}-1}\left(y_{f}[k], \ldots, y_{f}[k-n]\right), \ldots,\left(y_{f}[k], \ldots, y_{f}[k-n]\right),\right.} \\
& \left.D^{n_{c}}\left(u_{f}[k], \ldots, u_{f}[k-n]\right), \ldots,\left(u_{f}[k], \ldots, u_{f}[k-n]\right)\right), \\
\phi_{2}^{T}(k, \tau)= & \left(D^{n_{c}}\left(u_{f}((k-1) T+\tau), \ldots, u_{f}((k-n) T+\tau)\right), \ldots,\right. \\
& \left(u_{f}((k-1) T+\tau), \ldots, u_{f}((k-n) T+\tau)\right), \\
\theta_{0}^{T}= & \left(f_{1}-\alpha_{1}, f_{2}-\alpha_{2}, \ldots, f_{n}-\alpha_{n}, \beta_{0}, \beta_{1}, \ldots, \beta_{n}\right)^{T}, \\
\theta_{1}^{T}= & {\left[\left(\beta_{0}^{10}, \ldots, \beta_{0}^{1 n}\right), \ldots,\left(\beta_{n_{c}}^{10}, \ldots, \beta_{n_{c}}^{1 n}\right), \ldots,\left(\beta_{0}^{20}, \ldots, \beta_{0}^{2 n}\right), \ldots,\left(\beta_{n_{c}}^{20}, \ldots, \beta_{n_{c}}^{2 n}\right)\right), } \\
\theta_{2}^{T}(\tau)= & {\left[\left(\beta_{0}^{31}(\tau), \ldots, \beta_{0}^{3 n}(\tau)\right), \ldots,\left(\beta_{n_{c}}^{31}(\tau), \ldots, \beta_{n_{c}}^{3 n}(\tau)\right)\right.}
\end{aligned}
$$

for all $t \in[k T,(k+1) T)$ and $\tau \in[0, T)$.

Remark 2.4. Note also that the parameter vectors in (2.12a) contain sub-vectors of parameters normalized in $T$ so that the relations in (2.10) and the various ones in (2.11) and (2.12) are identical. The associated descriptions involving whole parameterizations under the integral symbol will then makes possible the statement of the estimation scheme of Section 3. The first motivation to adopt such an integral-type description is the presence of the regressor $\phi_{2}(\cdot)$ which depends on time during the inter-sample period. The second and main motivation to adopt the integral-type parameterization (2.12) to describe (2.11) is its convenience for analysis in the adaptive case then discussed in Section 3. The reason is that the statement of an adaptation algorithm involving a covariance matrix for the whole parameter vector depending on a dummy time argument which belongs to the inter-sample period facilitates the use of a Lyapunov's-like function. Such a function is defined with the parametrical error and then used for robust stability and convergence 
analysis. Thus, the subsequent stability and robustness analysis of the adaptive system is simpler compared to a potential choice of separate adaptation gains for $\theta_{2}(\cdot)$ and the remaining parameter sub-vectors in $(2.12 \mathrm{a})$.

2.3. Problem statement. The problem to be addressed in the sequel is the adaptive control of an hybrid system, whose nominal state-space representation is (2.1), in the presence of bounded noise and a standard class of unmodeled dynamics (see [14] for purely continuous plants and [8] for hybrid systems of first-order continuous-time parts) when the plant parameters are not completely known. The class of plants (i.e., controlled objects) (2.1) dealt with in this paper is that consisting of coupled continuous and digital linear subsystems operated with continuous-time inputs, which are almost everywhere continuous functions with eventual bounded discontinuities at sampling instants, and its corresponding sampled values at sampling instants. It is assumed that only input and output measurements, as well as an upper-bounding function of the contribution of the uncertainties to the output, are available (i.e., the state vector might be not available for measuring) so that the system described by the input-output description (2.10) (or, equivalently, (2.11) and (2.12)). The control objective is the asymptotic tracking of any bounded reference signal $y^{*}(t)$ with prescribed closed-loop pole-placement in the ideal case of nominal plant with known parameters. The parameter estimation scheme is of a continuous-time nature for all the plant parameters including those associated with the digital subsystem. In the nonideal case of unknown parameters, the objective is relaxed to the achievement of robust closed-loop stability. In the following, the subsequent set of assumptions are made:

Assumption 2.5. The orders of the nominal sub-states $n_{c}$ and $n_{d}$ of (2.1) are known.

Assumption 2.6. $\alpha(D)$ and $\beta(D)$ are coprime polynomials.

Assumption 2.7. The disturbance signal $\eta_{f}(t)$, due to unmodeled dynamics and bounded noise, is over-bounded by a measurable function as follows:

$$
\left|\eta_{f}(t)\right| \leq \gamma(t):=\varepsilon \rho(t)+\varepsilon_{0}
$$

for all $t \in[k T,(k+1) T)$ and all integer $k \geq 0$ with

$$
\begin{gathered}
\rho(t):=\sup _{0 \leq \tau \leq T}\left(\sup _{0 \leq \tau^{\prime} \leq t}\left(e^{-\sigma_{0}\left(t-\tau^{\prime}\right)}\|z(t, \tau)\|_{2}\right)\right), \\
z(t, \tau)=\left(\bar{\phi}_{0}^{T}(t), \bar{\phi}_{1}^{T}[k], \phi_{2}^{T}(k, \tau)\right)^{T},
\end{gathered}
$$

where $\sigma_{0} \geq 0, \varepsilon \geq 0$, and $\varepsilon_{0} \geq 0$ are known real constants, and the regressors with superscripts bar in (2.14b) are obtained from the corresponding ones in (2.12) by deleting their components associated with the successive $n_{c}$ th time-derivatives.

Assumption 2.5 is standard in most of the literature on adaptive control of continuous and discrete systems (see, for instance, $[3,6,8,16,29]$ ). Note from Assumption 2.6 
that the nominal plant is controllable while it is not assumed to be inversely stable (see Lemma 2.1 and Remark 2.2). This is an important feature concerning the synthesis of pole-placement type controllers which cancel the plant zeros. Note also that the coprimeness of the polynomials $\alpha(D)$ and $\beta(D)$ ensures that $\left(\alpha(D)-\beta_{10}(D), \beta(D)+\beta_{20}(D)\right)$ are coprime polynomials. Then, the input-output description (2.9) "at" sampling instants admits controllable state-space realizations if $(2.4 \mathrm{a})$ is controllable; that is, if the controllability holds "in-between" sampling instants. The knowledge of the over-bounding functions for unmodeled dynamics is also usual (see, for instance, [8, 21, 38]). The class of unmodeled dynamics considered is described in Assumption 2.7. Also, the constants $\varepsilon$ and $\varepsilon_{0}$ of (2.13) have been assumed unknown in [14] while incorporating their estimations to an extended estimation scheme. However, both simplifications are omitted in this paper since they are not essential for the topic described while Assumptions 2.5 and 2.7, as stated, facilitate the clarity of presentation. On the other hand, it can be proved as a mathematical result that Assumption 2.7 is fulfilled when the filtered unmodeled dynamics contribution to the output $\eta_{f}(t)$ is related to the input by exponentially stable transfer functions. If additive and multiplicative transfer functions associated with unmodeled dynamics in (2.10) are exponentially stable then $\sigma_{0}>0$.

\section{Estimation scheme and adaptive controller}

In the following, the estimation scheme for the input-output descriptions (2.11) and (2.12) of the current hybrid plant involving unmodeled dynamics and/or bounded noise is given. The algorithm involves the use of a relative dead zone to prevent the system against possible instability caused by uncertainties.

3.1. Estimation scheme. If the parameters are replaced by their estimates, denoted with superscripts "hat," then (2.11) becomes

$$
\begin{aligned}
y(t)= & F(D) y_{f}(t)=(F(D)-\hat{\alpha}(D, t)) y_{f}(t)+\hat{\beta}(D, t) u_{f}(t) \\
& +\sum_{i=1}^{n} \int_{0}^{T} \hat{\beta}_{3 i}(D, t, \tau) u_{f}[(k-i) T+\tau] d \tau+c_{F}^{T} e^{A_{F} t} \hat{\bar{x}}^{*}(0, t)+e(t) \\
= & \int_{0}^{T} \phi^{T}(k, \tau) \hat{\theta}(t, \tau) d \tau+e(t)
\end{aligned}
$$

for $t \in[k T,(k+1) \tau)$ where $\hat{\alpha}(D, t), \hat{\beta}(D, t)$, and $\hat{\bar{x}}^{*}(0, t)$ being the estimates of $\alpha(D)$, $\beta(D)$, and $\hat{\bar{x}}^{*}(0)$, respectively, and

$$
e(t)=y(t)-\int_{0}^{T} \phi^{T}(k, \tau) \hat{\theta}(t, \tau) d \tau=-\int_{0}^{T} \phi^{T}(t, \tau) \tilde{\theta}(t, \tau) d \tau+\eta_{f}(t)
$$

is the estimation error and $\tilde{\theta}(t, \tau)=\hat{\theta}(t, \tau)-\theta(\tau)$ for $\tau \in[0, T)$ and all $t \geq 0$ is the parametrical error. The various estimates are updated by using (3.2) with the subsequent 
continuous-time least-squares algorithm with forgetting factor with relative dead zone:

$$
\begin{gathered}
\dot{\hat{\theta}}(t, \tau)=b(t) P(t, \tau) \phi(t, \tau) e(t) ; \quad \hat{\theta}(0, \tau)=\hat{\theta}^{0}(\tau), \\
\dot{P}(t, \tau)=\lambda(t) P(t, \tau)-b(t) P(t, \tau) \phi(t, \tau) \phi^{T}(t, \tau) P(t, \tau) ; \quad P(0, \tau)=P^{T}(0, \tau)=P^{0}(\tau)>0, \\
b(t)=\frac{\alpha s(t)}{1+p(t)} ; \quad p(t)=\sup _{0 \leq \tau \leq T}\left(\lambda_{\max }(P(t, \tau))\right) \sup _{0 \leq \tau \leq T}\left(\|\phi(t, \tau)\|^{2}\right),
\end{gathered}
$$

for $\tau \in[0, T)$ and all $t \geq 0$ with $\bar{\sigma}>0, k_{\sigma}>1$, and $\zeta>1$ being design constants. Note that this least-squares algorithm is not of a modified type as that proposed for the discrete parameters. However, the forgetting factor is fixed to zero if the covariance matrix norm exceeds a prefixed value (see (3.3b), (3.3d), and (3.3e)). This guarantees the boundedness of the norm of the covariance matrix.

3.2. Control law and adaptive pole-placement. The following technical assumption is made for solvability of the controller synthesis.

Assumption 3.1. The estimates $\hat{\alpha}(D, t)$ and $\hat{\beta}(D, t)$ of the polynomials $\alpha(D)$ and $\beta(D)$ are coprime for all time and their limits as $t \rightarrow \infty$ are also coprime.

Assumption 3.1 is only made to facilitate the paper exposition without cumbersome calculations. In fact, the incorporation of a estimation modification scheme based on the use of a hysteresis switching function would avoid directly the singular and near singular cases in the synthesis of the adaptive controller by only requiring coprimeness of the true polynomials (Assumption 2.6). The possible existence of singularities in the diophantine equations associated with the adaptive controller synthesis can be avoided by using a modified estimated plant model whose controllability is guaranteed through the modification estimation algorithm. Such a modification can be obtained via a hysteresis switching function which guarantees that the absolute controllability index of the modified estimated model is positively bounded from below for all time. See [8] for a simple hybrid system where the couplings between continuous and sampled signals appear 
directly in the input-output model (2.11) and (2.12). The same technique was also used in adaptive control of time-delay systems, $[9,38]$. The avoidance of singularities in the estimated model is made possible since Assumption 2.6 guarantees the solvability of the diophantine equation associated with the controller synthesis for the nominal input-output models obtained in Lemma 2.1 in the case of known plant parameters.

The controllability of the modified estimated plant model avoids, in addition, the need for the assumption of signal persistent excitation (or the injection of auxiliary signals fulfilling such an hypothesis) as well as alternatively the use of the alternative which requires the knowledge of appropriate convex parameter sets, where the model is uniformly stabilizable, to be used for projection of the estimates [33]. An output expression equivalent to (2.11) (2.12), and (3.2) which is useful to discuss the adaptive pole-placement problem is:

$$
\begin{aligned}
\left(\int_{0}^{T} \hat{\alpha}^{\prime}(D, t, \tau) d \tau\right) y_{f}(t)= & \left(\int_{0}^{T} \hat{\beta}^{\prime}(D, t, \tau) d \tau\right) u_{f}(t)+\sum_{i=0}^{n} \int_{0}^{T} \hat{\beta}_{1 i}^{\prime}(D, t, \tau) y_{f}[(k-i) T+\tau] d \tau \\
& +\sum_{i=0}^{n} \int_{0}^{T} \hat{\beta}_{2 i}^{\prime}(D, t, \tau) u_{f}[(k-i) T+\tau] d \tau \\
& +\sum_{i=1}^{n} \int_{0}^{T} \hat{\beta}_{3 i}(D, t, \tau) u_{f}[(k-i) T+\tau] d \tau \\
& +\int_{0}^{T} c_{F}^{T} e^{A_{F} t} \hat{\bar{x}}^{*^{\prime}}(0, t, \tau) d \tau+e(t)
\end{aligned}
$$

for $t \in(k T,(k+1) T)$ and all integer $k \geq 0$ where $\hat{\alpha}(D, t)=\int_{0}^{T} \hat{\alpha}^{\prime}(D, t, \tau) d \tau, \hat{\beta}(D, t)=$ $\int_{0}^{T} \hat{\beta}^{\prime}(D, t, \tau) d \tau$ and $\hat{\bar{x}}^{*}(0, t)=\int_{0}^{T} \hat{\bar{x}}^{*^{\prime}}(0, t, \tau) d \tau$ with $\hat{\alpha}^{\prime}(D, t, \tau), \hat{\beta}^{\prime}(D, t, \tau), \hat{\beta}_{1 i}^{\prime}(D, t, \tau)$, $\hat{\beta}_{2 i}^{\prime}(D, t, \tau)$ and $\hat{\bar{x}}^{\prime}(0, t, \tau)$ being the estimates of $\alpha(D) / T, \beta(D) / T, \beta_{1 i}(D) / T, \beta_{2 i}(D) /$ $T \beta_{1 i}(D, t, \tau)$ and $\bar{x}^{*}(0) / T$, respectively, for $\tau \in[0, T)$ whose coefficients are obtained from the components of $\hat{\theta}(t, \tau)$ calculated in (3.4). Now, rewrite (2.11b), or equivalently (3.4), in a more compact way as follows in order to later establish the adaptive control law:

$$
\hat{\alpha}(D, t) y_{f}(t)=\hat{\beta}(D, t) u_{f}(t)+\hat{v}(t)+e(t)
$$

for $t \in[k T,(k+1) T)$, where

$$
\begin{aligned}
\hat{v}(t)= & \hat{v}[k]+\hat{\tilde{v}}(t)=\sum_{i=0}^{n}\left(\hat{\beta}_{1 i}(D, t) y_{f}[k-i]+\hat{\beta}_{2 i}(D, t) u_{f}[k-i]\right) \\
& +\sum_{i=1}^{n} \int_{0}^{T} \beta_{3 i}(D, t, \tau) u_{f}[(k-i) T+\tau] d \tau+c_{F}^{T} e^{A_{F} t} \hat{\bar{x}}^{*}(0, t) \\
= & \int_{0}^{T} \phi_{1}^{T}[k] \hat{\theta}_{1}(t, \tau) d \tau+\int_{0}^{T} \phi_{2}^{T}(k, \tau) \hat{\theta}_{2}(t, \tau) d \tau+\int_{0}^{T} c_{F}^{T} e^{A_{F} t} \hat{\bar{x}}^{\prime}(0, t, \tau) d \tau
\end{aligned}
$$


with

$$
\begin{aligned}
\hat{v}[k]= & \hat{\alpha}(D, k T) y_{f}[k]-\hat{\beta}(D, k T) u_{f}[k]-e[k] \\
\hat{\tilde{v}}(t)= & \sum_{i=0}^{n}\left[\hat{\beta}_{1 i}(D, t)-\hat{\beta}_{1 i}(D, k T)\right] y_{f}[k-i]+\sum_{i=0}^{n}\left[\hat{\beta}_{2 i}(D, t)-\hat{\beta}_{2 i}(D, k T)\right] u_{f}[k-i] \\
& +\sum_{i=1}^{n} \int_{0}^{T}\left[\hat{\beta}_{3 i}(D, t, \tau)-\hat{\beta}_{3 i}(D, k T, \tau)\right] u_{f}[(k-i) T+\tau] d \tau \\
& +c_{F}^{T} e^{A_{F} t}\left(\hat{\bar{x}}^{*}(0, t)-\hat{\bar{x}}^{*}(0, k T)\right) \\
= & \int_{0}^{T}\left(\phi_{1}^{T}[k]\left(\hat{\theta}_{1}(t, \tau)-\hat{\theta}_{1}(k T, \tau)\right)+\phi_{2}^{T}(k, \tau)\left(\hat{\theta}_{2}(t, \tau)-\hat{\theta}_{2}(k T, \tau)\right)\right) d \tau \\
& +\int_{0}^{T} c_{F}^{T} e^{A_{F} t}\left(\hat{\bar{x}}^{*}(0, t, \tau)-\hat{\bar{x}}^{*}(0, k T, \tau)\right) d \tau
\end{aligned}
$$

for all $t \in[k T,(k+1) T)$ and all integer $k \geq 0$. The use of the driving signal $\hat{v}(t)=\hat{v}[k]+$ $\hat{\tilde{v}}(t)$ in (3.5) allows to characterize the output in the inter-sample period by using the disturbance signal $\hat{\tilde{v}}(t)$ with respect to the values of $\hat{v}[k]$ at sampling instants. Such a characterization is a key point in ensuring the adaptive robust stability of Theorem 4.2 in Section 4 below. Note that in the current context of this paper, the objective of the adaptive technique is related to the achievement of the stability of a dynamic feedback system rather than to numerical stability or stability of systems described by partial differential equations $[20,35]$. The filtered control input "at" and "in-between" sampling instants is generated from the time-varying difference equation:

$$
L(D, t) u_{f}(t)=R(D, t)\left(y_{f}^{*}(t)-y_{f}(t)+Q(D, t) \hat{v}[k]\right),
$$

where $y_{f}^{*}(t)=(1 / F(D)) y^{*}(t)$ is the filtered reference signal, $Q(D, K T)$ is a rational function and $Q(D, t)=0$ for $t \neq k T$, otherwise, with polynomials $L(D, t)=1+\sum_{i=1}^{n_{c}+1} l_{i}(t) D^{n_{c}+1-i}$ and $R(D, t)=\sum_{i=0}^{n_{c}} r_{i}(t) D^{n_{c}-i}$ for all $t \geq 0$. Note that the unfiltered control $u(t)=F(D) u_{f}(t)$ signal is well-posed since $u_{f}(t)$ is a $n_{c}$ th continuously differentiable function which is synthesized from (3.8). The controller synthesis problem, that is, the synthesis of $Q, L$, and $R$, is solved as follows:

3.2.1. "At" sampling instants ( $t=k T$, all integer $k \geq 0)$. The polynomials $L(D, k T)$ and $R(D, k T)$ are uniquely calculated from the diophantine equation

$$
\hat{\alpha}(D, k T) L(D, k T)+\hat{\beta}(D, k T) R(D, k T)=A^{*}(D)
$$

and $Q(D, k T)$ in $(3.8)$ is calculated from

$$
K[k]=\operatorname{Max}\left(1+\sum_{j=1}^{n_{c}}\left|a_{j}^{*}\right| ; 1+\sum_{j=1}^{n_{c}+1}\left|l_{j}(k T)\right|\right) ; \quad Q(D, k T)=\frac{K[k] A_{v}^{*}(D)-L(D, k T)}{\hat{\beta}(D, k T) R(D, k T)}
$$


for given strictly Hurwitz monic polynomials $A^{*}(D)$ and $A_{v}^{*}(D)$ of respective degrees $n_{c}$ and $2 n_{c}-2$ if $d=d_{c}+d_{d} \neq 0$, and $\operatorname{deg}\left(A_{v}^{*}\right)=2 n_{c}-1$, otherwise, and of respective stability abscissas $\left(-\rho^{*}\right)<0$ and $\left(-\rho_{v}^{*}\right)<0 . K[k] \geq 1$ is an adjustable gain which is up-dated on-line for stabilization purposes as a part of the synthesis of the adaptive controller. The role of such a gain is to avoid a large forcing term depending of the filtered plant output at sampling instants which is generated by the couplings from the dynamics at sampling instants to the dynamics within the inter-sample period as reflected in Lemma 2.1. More technical details about the specific role played by such a time-varying gain in the closedloop stabilization will be then addressed in the comments about the main stability and robustness results of Section 4.2 and the proof of the main result in Appendix B. See the structures of (2.1) and the input-output description of the filtered plant in Lemma 2.1 for more details. Note from (3.5), (3.8), and (3.9) that the closed-loop system at sampling instants is described by

$$
A^{*}(D) y_{f}[k]=\hat{\beta}(D, k T) R(D, k T) y_{f}^{*}[k]+K[k] A_{v}^{*}(D) \hat{v}[k]+L(D, k T) e[k]
$$

which can be rewritten as

$$
\hat{v}[k]=\frac{1}{K[k] A_{v}^{*}(D)}\left\{A^{*}(D) y_{f}[k]-\hat{\beta}(D, k T) R(D, k T) y_{f}^{*}[k]-L(D, k T) e[k]\right\} .
$$

The same expression is obtained after substituting the control law (3.8) for $t=k T$ with $\hat{\tilde{v}}[k]=0$ into $(3.5)$ by using $(3.9 \mathrm{a})$ and $(3.9 \mathrm{~b})$.

3.2.2. "In-between" sampling instants $(t \neq k T$, all integer $k \geq 0)$. Within the inter-sample intervals, $Q(D, t)=0$ and $L(D, t)$ and $R(D, t)$ are uniquely calculated from the diophantine equation:

$$
\hat{\alpha}(D, t) L(D, t)+\hat{\beta}(D, t) R(D, t)=A^{*}(D) .
$$

The substitution of (3.11) into (3.5) for $t \neq k T$ yields directly

$$
\begin{aligned}
\hat{\alpha}(D, t) y_{f}(t)= & \hat{\beta}(D, t) u_{f}(t)+\frac{1}{K[k] A_{v}^{*}(D)} \\
& \times\left\{A^{*}(D) y_{f}[k]-\hat{\beta}(D, k T) R(D, k T) y_{f}^{*}[k]-L(D, k T) e[k]\right\}+\hat{\tilde{v}}(t)+e(t) \\
= & \hat{\beta}(D, t) u_{f}(t)+\hat{v}[k]+(\hat{\hat{v}}(t)+e(t))
\end{aligned}
$$

"in-between" sampling instants with the associated closed-loop equation obtained by substituting the control law (3.8) into (3.13)

$$
\begin{aligned}
A^{*}(D) y_{f}(t)= & \hat{\beta}(D, t) R(D, t) y_{f}^{*}(t)+\frac{L(D, t)}{K[k] A_{v}^{*}(D)} \\
& \times\left\{A^{*}(D) y_{f}[k]-\hat{\beta}(D, k T) R(D, k T) y_{f}^{*}[k]-L(D, k T) e[k]\right\} \\
& +L(D, t)[\hat{\tilde{v}}(t)+e(t)\rfloor \\
= & \hat{\beta}(D, t) R(D, t) y_{f}^{*}(t)+L(D, t)(\hat{v}(t)+e(t))
\end{aligned}
$$


for $t \in[k T,(k+1) T)$ and all integer $k \geq 0$. Since $A^{*}(D)$ and $\hat{\alpha}(D, t)$ are monic polynomials, $L(D, t)$ is monic. The combined filtered controller and plant equations (3.8) and (3.13) within the inter-sample period can be described through the following $\left(2 n_{2}+1\right)$ th order auxiliary extended system

$$
\begin{aligned}
\dot{x}(t)= & A(t) x(t) \\
& +\mu_{1}\left(\frac { 1 } { K [ k ] A _ { v } ^ { * } ( D ) } \left(A^{*}(D) y_{f}[k]-\hat{\beta}(D, k T) R(D, k T) y_{f}^{*}[k]\right.\right. \\
& \quad-L(D, k T) e[k])+\hat{\tilde{v}}(t)+e(t)) \\
= & A(t) x(t)+\mu_{1}\lfloor\hat{v}[k]+(\hat{\tilde{v}}(t)+e(t))\rfloor,
\end{aligned}
$$

where with $\mu_{(\cdot)}$ is the $(\cdot)$ th unity Euclidean vector in $\mathbf{R}^{2 n_{c}+1}$, and

$$
\begin{aligned}
& x(t)=\left(D^{n_{c}-1} y_{f}(t), \ldots, D y_{f}(t), y_{f}(t), \ldots, D^{n_{c}} u_{f}(t), \ldots, D u_{f}(t), u_{f}(t)\right)^{T} \\
& A(t)=\left[\begin{array}{cccccccc}
-\hat{\alpha}_{1}(t) & -\hat{\alpha}_{2}(t) & \ldots, & \hat{\alpha}_{n_{c}}(t) & \hat{\beta}_{0}(t) & \hat{\beta}_{1}(t) & \ldots, & \hat{\beta}_{n_{c}}(t) \\
1 & 0 & \ldots, & 0 & 0 & \ldots & \ldots & 0 \\
0 & \vdots & \ddots & 0 & \vdots & \vdots & \vdots & \vdots \\
0 & \ldots & \ldots & 1 & 0 & \ldots & \ldots & 0 \\
-r_{0}(t) & -r_{1}(t) & \ldots & -r_{n_{c}}(t) & -l_{1}(t) & -l_{2}(t) & \ldots-l_{n_{c}}(t) & -l_{n_{c}+1}(t) \\
0 & \ldots & \ldots & 0 & 1 & 0 & \ldots & 0 \\
\vdots & \vdots & \ddots & \vdots & 0 & \vdots & \vdots & \vdots \\
\vdots & \vdots & \vdots & \vdots & \vdots & \vdots & \ddots & 0 \\
0 & \ldots & \ldots & 0 & 0 & \ldots & \ldots & 1
\end{array}\right] .
\end{aligned}
$$

Remark 3.2. The interpretation of the diophantine equation (3.9a) is direct from the description (2.9) of the filtered plant at sampling instants. Assume that the objective polynomial prefixing the stable suited closed-loop poles is $A^{*}(D)$ for the diophantine equation:

$$
\left\lfloor\hat{\alpha}(D, k T)-\hat{\beta}_{10}(D, k T)\right\rfloor L^{\prime}(D, k T)+\left\lfloor\hat{\beta}(D, k T)+\hat{\beta}_{20}(D, k T)\right\rfloor R^{\prime}(D, k T)=A^{*}(D)
$$

and the controller polynomials are

$$
L^{\prime}(D, k T)=L(D, k T)+\Delta L(D, k T) ; \quad R^{\prime}(D, k T)=R(D, k T)+\Delta R(D, k T)
$$

with the pair $(L(D, k T), R(D, k T))$ satisfying uniquely (3.9a). Thus, the incremental polynomials $\Delta L(D, k T)$ and $\Delta R(D, k T)$ are the unique solution to

$$
\begin{gathered}
\left(\hat{\alpha}(D, k T)-\hat{\beta}_{10}(D, k T)\right) \Delta L(D, k T)+\left(\hat{\beta}(D, k T)+\hat{\beta}_{20}(D, k T)\right) \Delta R(D, k T) \\
=A^{*}(D)+\hat{\beta}_{10}(D, k T) L(D, k T)-\hat{\beta}_{20}(D, k T) R(D, k T)
\end{gathered}
$$


with $\operatorname{deg}(\Delta L)=\operatorname{deg}(\Delta R+1)=n_{c}+1$ and $L(D, k T)$ and $R(D, k T)$ being the solution to (3.9a). The role of those controller incremental polynomials is related to the change of parameterization from (2.4a) to (2.9); that is, at sampling instants with respect to the model description within the intersample period.

Remark 3.3. The controllability of the estimated plant model is assumed for all time and as time tends to infinity. Assumption 3.1 is made to avoid cumbersome a posteriori analytical estimation scheme modifications that guarantee the avoidance of the singular and near singular cases when solving the diophantine equation for the synthesis of the adaptive controller. Such modifications together with the analytical proofs of convergence and stability can be directly extended from their counterparts in the classical continuoustime and discrete cases (see $[8,14,33])$.

In particular, the nearly singular case is avoided in [33] by assuming that the adaptive scheme is asymptotically stabilizable as a time-varying system. On the other hand, singularities are avoided in $[17,31,38]$, and thus the controllability of the estimation model is guaranteed, by using additional input impulses at certain time instants. Estimation modification schemes based upon the properties of the covariance matrix were recently proposed for purely continuous-time and discrete-time linear plants as well as for a class of first-order hybrid systems [8]. In addition, that technique allows the relaxation of the assumption of plant inverse stability since the singularities are avoided when solving the diophantine equations associated with the controller synthesis. It also avoids either the use of excitation probing signals as in $[17,31]$ or projection of the estimates on a known convex region to guarantee the system's stabilizability, [14, 38]. The avoidance of the two above mentioned related techniques is advantageous since they cause either implementation drawbacks or require extra "a priori" knowledge on the plant parameters, $[8,14,32,33]$.

Remark 3.4. Note that the control law (3.8) involves the use of a compensating signal $\hat{v}[k]$ and an extra sampling-dependent gain $K[k]$ at sampling instants. Their role is to compensate for the presence of the coupling $s$ from the input/output samples and from the input integral to the time-differential description causes the presence of an additive signal in the input-output plant description (see Lemma 2.1 and (3.5)).

\section{Stability and robustness results}

4.1. Boundedness and convergence properties of the estimation scheme. The properties of the estimation algorithm is given in the subsequent result, which is proved in Appendix B, that is independent of the control law (3.8) or of any other possible control law used.

LemmA 4.1. The estimation algorithm (3.3) has the following properties:

(i) $b(t) \rightarrow 0, b(t) \eta_{f}^{2}(t) \rightarrow 0, b(t) \gamma^{2}(t) \rightarrow 0, b(t)\left(e(t)-\eta_{f}(t)\right)^{2} \rightarrow 0, b(t)\left(|e(t)|-\left|\eta_{f}(t)\right|\right)^{2}$ $\rightarrow 0$ as $t \rightarrow \infty$.

(ii) $\hat{\theta}(t, \tau), P(t, \tau)$, and $P^{-1}(t, \tau)$ are uniformly bounded in $\tau \in[0, T)$ for all $t \geq 0$ and $\hat{\theta}(t, \tau)$ converges asymptotically to a finite limit as $t \rightarrow \infty$ for all $\tau \in[0, T)$. 
(iii) $\dot{\hat{\theta}} \in L_{2} \cap L_{\infty}$ for all $\tau \in[0, T)$. Furthermore, $b^{1 / 2}|e| \in L_{2} \cap L_{\infty}, b^{1 / 2}\left|\eta_{f}\right| \in L_{2} \cap L_{\infty}$, $b^{1 / 2}|\gamma| \in L_{2} \cap L_{\infty}, b^{1 / 2}\left|e-\eta_{f}\right| \in L_{2} \cap L_{\infty}, b^{1 / 2}\left|\left(|e|-\left|\eta_{f}\right|\right)\right| \in L_{2} \cap L_{\infty}$ and $b(e-$ $\gamma)^{2} \in L_{1} \cap L_{\infty}$.

The properties of asymptotic convergence of the estimates to a finite limit in Lemma 4.1(ii) as well as the integrability properties of Lemma 4.1(iii) are crucial in the proof of the main stability and robustness result (Theorem 4.2 below). In addition, Lemma 4.1 leads to simpler particular properties in the perfectly modeled case of absence of bounded noise and unmodeled dynamics. For instance, in that case, Lemma 4.1(i) yields the asymptotic convergence of the prediction error to zero and Lemma 4.1(ii) yields the square-integrability of the prediction error on $[0, \infty)$. The properties of boundedness, convergence to finite limits and integrability given in Lemma 4.1 will be then used in the proof of the main stability and robustness result of the closed-loop system which is given in the next subsection.

\subsection{Robust closed-loop stability. The following result is proved in Appendix B.}

Theorem 4.2 (main stability and robustness result). The following propositions hold:

(i) Assume that $A_{v}^{*}(D)$ is chosen so that $\rho_{v}^{*}>\left(1+\rho_{0}\right) / \rho^{*}$ for some design real positive constant $\rho_{0}$. Assume also that the unmodeled dynamics is sufficiently small in Assumption 2.7 satisfying $\varepsilon<\left(\rho^{*} \rho_{v}^{*}-1-\rho_{0}\right) / 2 \zeta\left(\rho_{v}^{*}+1\right)$. Assume also that the controller synthesis is made by solving (3.9) and (3.12). Thus, the closed-loop adaptive system is globally stable so that all the filtered input and output signals in the loop as well as their time-derivatives up-till order $n_{c}$ and the plant unfiltered input and output signals are bounded "at" and "in-between" sampling instants for any bounded initial conditions.

(ii) If, in addition to the assumptions in (i), $\varepsilon_{0}=0$ and, furthermore, $y^{*}(t)$ converges asymptotically to zero then $D^{i} y_{f}(t) \rightarrow 0 ; D^{i} u_{f}(t) \rightarrow 0$ asymptotically as $t \rightarrow \infty ; i=0,1, \ldots, n_{c}$ and also $y_{f}(t) \rightarrow 0$ and $u_{f}(t) \rightarrow 0$ asymptotically as $t \rightarrow \infty$.

The proof is performed by using the auxiliary dynamic system (3.15) whose statevector is defined by the filtered plant input and output and a set of their successive timederivatives up-till appropriate orders. Such a system is obtained from the external inputoutput description of Lemma 2.1 for the plant plus the control and it is driven by the adaptation error and the contribution of the unmodeled dynamics to the output. Firstly, the unforced auxiliary system is proved to be globally uniformly exponentially stable. Then, the global stability of the forced auxiliary system is guaranteed provided that the size of the unmodeled dynamics is sufficiently small compared with the stability degree of the polynomial $A^{*}(D) \cdot A_{v}^{*}(D)$ (see Theorem 4.2). As a result, that of the closed-loop adaptive system is also guaranteed. A main tool used in the proof of stability is Gronwall's lemma whose manipulation requires a small gain-type condition, $[13,21,40]$. The filtered plant output and the prediction error "at" sampling instants generate forcing signals for the auxiliary system (3.15) which contribute to drive the plant output during the inter-sample periods. The time-varying gain $K[k]$ is updated at sampling instants by using (3.9b) so that the filters in the right-hand side of (3.15) have sufficiently small gains. That strategy guarantees robust stability for small sizes of the unmodeled dynamics. An 
intermediate result which also holds and is used in the proof of Theorem 4.2 is the asymptotic convergence to zero of the signal $\hat{\tilde{v}}(t)$ defined in $(3.7 \mathrm{c})$ since the estimates converge to finite limits.

\section{Identification issues}

The identification of parameters requires that the uncontrolled plant be stable and perfectly modeled and stable in the absence of disturbances and, furthermore, the input is persistently excited. The extra hypotheses are:

Assumption 5.1. The nominal extended discrete system $(\cdot)$ which describes (2.1) at sampling instants is controllable from the individual input sequences $u[k]$ and $v[k]$ and exponentially stable.

Assumption 5.2. The scalar input $u(t)$ is bounded for all time and, in addition, $m[k]=$ $\left(U[k], v^{T}[k]\right)^{T} \cdot U(t-k T)$, with $U(t)$ denoting the unity step function at $t=0$, is persistently excited of order $n_{\phi e}=\max \left(6 n,(3 n+2) \cdot n_{c}\right)$ (see, for instance [32]), that is, there exist positive real constants $S, \gamma_{0}$ and $\gamma_{1} \geq \gamma_{0}$ such that $\infty>\gamma_{1} I \geq(1 / S)\left(\sum_{j=k}^{k+n_{\phi e}} m[j] \cdot m^{T}[j]\right) \geq$ $\gamma_{0} I>0$ for all integer $k \geq 0$.

Basically, those assumptions allow to transfer the property of persistent of excitation from the discretized input (Assumption 5.2) to the filtered input and then to the regressor in absence of controller (open-loop case) provided that the discretized plant is exponentially stable (Assumption 5.1), $[1,10]$. Note that these extra assumptions allow to guarantee that the true parameters have the appropriate nominal dimension and that the bounded estimates converge asymptotically to their true values as time tends to infinity as it can be deduced using Lemma 4.1 if the contribution of the unmodeled dynamics to the output is zeroed (i.e., for the open-loop nominal plant case). The proofs are omitted for the shake of brevity of the manuscript. The properties of asymptotic convergence of the estimates to a finite limit in Lemma 4.1(ii) as well as the integrability properties of Lemma 4.1(iii) are crucial in the proof of the main stability and robustness result (Theorem 4.2 below). Note that, since no unmodeled dynamics is present, the time-varying gain of the estimation scheme $b(t)$ is unity for all time. This implies that the estimation is never frozen by the estimation scheme and, in the case that this circumstance be known by the designer the relative dead-zone could be removed from the estimation. In addition, Lemma 4.1 leads to simpler particular properties in the perfectly modeled case of absence of bounded noise and unmodeled dynamics. For instance, in that case, Lemma 4.1(i) yields the asymptotic convergence of the prediction error to zero and Lemma 4.1(iii) yields the square-integrability of the prediction error on $[0, \infty)$. The properties of boundedness, convergence to finite limits and integrability given in Lemma 4.1 have to be invoked in the proof of the following result for the nominal hybrid plant.

Theorem 5.3. Assume that Assumptions 2.5, 2.6, 5.1, and 5.2 hold and that the hybrid plant is noise-free and perfectly modeled. Thus, the regressor of the parameter estimation algorithm (3.3) is persistently excited. In addition to the properties of Lemma 4.1, the parameter estimates converge asymptotically to their true values. 
The proof guideline is organized by using the properties of the estimates given in Lemma 4.1 and a set of auxiliary intermediate results concerning persistent of excitation of the various signals involved in the input-output descriptions given in Lemma 2.1 "at" and "in-between" sampling instants. The starting point is the persistence of excitation of the stepwise constant discrete values of the plant input "at" sampling instants. It is proved that such a property implies its persistence of excitation at all time. This also implies the persistence of excitation of the coupling sequence $\{v[k], k \geq 0\}$. Their filtered versions of all the above signals are also persistently excited under controllability and exponential stability of the filters. This also implies that the jointly filtered vector signal $\left(u_{f}^{T}[k], y_{f}^{T}[k], v_{f}^{T}[k]\right)^{T}$ is persistently excited. As a result, the regressor of the estimation scheme is persistently excited under controllability and exponential stability of the input-output descriptions of Lemma 2.1 "at" and "in-between" sampling instants what imply that an exponentially stable and controllable extended dynamic system, whose components include those of the state associated with the continuous-time description of Lemma 2.1(ii), is persistently excited.

\section{Conclusions and perspectives}

This paper has addressed the problem of robust adaptive control of a usual class of non inversely stable single-input single-output hybrid systems. The hybrid nature of the plant arises from the fact that it is simultaneously driven by a continuous-time input and its samples at sampling instants. As a result, the plant external description is given in terms of a continuous-time differential equation which is forced by the continuous time system input and by the samples of the input and output at sampling instants. The various signals are filtered by stable filters in order to improve the scheme's robustness against noise and unsuitable effects of unmodeled dynamics and also to achieve a relatively slow adaptation rate so as to improve the transient performances. The adaptive control scheme is based on pole-placement for the nominal case. All the system parameters, even those associated with the discrete signals, are estimated in a continuous-time fashion. This strategy avoids inherent discontinuities of the filtered plant input at sampling instants. The parameter estimation scheme possesses all the standard suitable properties of boundedness and convergence and consists of two parts. The estimation algorithm involves the use of a relative dead zone to prevent against instability caused by uncertainties. The dead zone freezes the parameter estimation when the size of the prediction error is small compared to the contribution to the uncertainties to the plant output. This ensures the solvability for all time of the time-varying diophantine equation associated with the synthesis of the adaptive controller. An important consequence is that the proposed scheme can be applied to plants with unstable zeros without losing the robust adaptive stabilization main objective.

Future work which remains open is the application of the given formalism to real problems where the plant consists of coupled discrete-time and continuous-time components and whose parameters are unknown and have to be estimated and to multivariable plants of the given class. Models within that class arise under the simultaneous presence of high frequency input signals with very slow ones which can be easily discretized. Another point to be addressed in this context is the separate estimation of the continuous-time and 
discrete-time parameters and to maintain separate continuous-time and discrete-time pole-placement objectives. That strategy would allow the use of different continuoustime and discrete-time reference models what would be of specific interest in problems when the sampling period is large.

\section{Appendices}

\section{A. Some preliminary technical proofs}

The two following preliminary technical results will be then used in the proof of Lemma 2.1.

Lemma A.1. (i) Consider the multi-input single-output time-invariant differential system

$$
A(D) v(t)=\sum_{i=1}^{p} B_{i}(D) u_{i}(t)
$$

subject to arbitrary bounded initial conditions $D^{i} v(0)=v_{i 0}(i=0,1, \ldots, s-1)$ with $v_{00}=$ $v(0)$ where $A(D)=D^{s}+A^{\prime}(D)=\sum_{i=0}^{s} a_{i} D^{s-i}$ and $B_{j}(D)=\sum_{i=0}^{r_{j}} b_{j i} D^{r_{j}-i}$ with $a_{0}=1$ and $\mathbf{b}_{r_{j}, j}$ and $\operatorname{deg}\left(B_{j}\right)=r_{j} \leq \operatorname{deg}(A)=s(j=1,2, \ldots, p)$. Thus, there is a $s \times\left(\sum_{j=1}^{p} r_{j}+s\right)$ constant real matrix $T_{z}$ for a state vector of any state-space realization of (A.1) fulfilling

$$
z(t)=T_{z}\left(D^{s-1} v(t), \ldots, D v(t), v(t), D^{r_{1}-1} u_{1}(t), \ldots, u_{1}(t), \ldots, D^{r_{p}-1} u_{p}(t), \ldots, u_{p}(t)\right)^{T} .
$$

(ii) Proposition (i) is extendible "mutatis-mutandis" to the case of difference equations $\bar{A}(q) v[k]=\sum_{i=1}^{p} \bar{B}_{i}(q) u_{i}[k] ; k \geq s$, where the polynomials $\bar{A}(q)$ and $\bar{B}(q)$ are defined similarly as $A(D)$ and $B(D)$ in the D-argument.

Proof. (i) First note from (A.1) that

$$
\dot{\bar{v}}=C_{A} \bar{v}+\sum_{j=1}^{p} \sum_{i=0}^{r_{j}} b_{i j} D^{r_{j}-i} u_{j}
$$

where $\bar{v}^{T}=\left(D^{s-1} v, D^{s-2} v, \ldots, D v, v\right)$ and $C_{A}=\left[\begin{array}{rc}-\mathbf{a}_{1}-\mathbf{a}_{2} \cdots-\mathbf{a}_{s-1} & -\mathbf{a}_{s} \\ 0 & \\ I_{s-1} & \vdots \\ 0\end{array}\right]$. Now, define the state vector

$$
\bar{x}=Q \bar{v}+\sum_{j=1}^{p} \sum_{i=1}^{r_{j}} q_{i j}^{\prime} D^{r_{j}-i} u_{j}
$$

with $Q=\left[q_{1}, \ldots, q_{s}\right]$ being any $s$-square real nonsingular matrix and $q_{i j}^{\prime}(j=1,2, \ldots, p, i=$ $\left.1,2, \ldots, r_{j}\right)$ being real $s$-vectors to be determined. The input is included in the definition of the state vector because the direct input-output transmission $d_{c}$ in (2.1a) may be nonzero and then $n_{c}$-first and higher-order time-derivatives can appear in the description of Lemma 2.1 and its extension in $[24,(13)]$. Taking time-derivatives in (A.4) while 
using (A.3) yields directly since $Q$ is nonsingular

$$
\begin{aligned}
\dot{\bar{x}}= & Q C_{A} \bar{v}+\sum_{j=1}^{p} Q b_{i j} D^{r_{j}} u_{j}+\sum_{j=1}^{p} q_{1 j}^{\prime} D^{r_{j}} u_{j}+\sum_{j=1}^{p} \sum_{i=1}^{r_{j}-1} q_{i+1, j}^{\prime} D^{r_{j}-i} u_{j} \\
= & Q C_{A} Q^{-1}\left(\bar{x}-\sum_{j=1}^{p} \sum_{i=1}^{r_{j}-1} q_{i j}^{\prime} D^{r_{j}-i} u_{j}\right)+\sum_{j=1}^{p} Q b_{0 j} D^{r_{j}} u_{j}+\sum_{j=1}^{p} \sum_{i=1}^{r_{j}-1} Q b_{i j} D^{r_{j}-i} u_{j} \\
& +\sum_{j=1}^{p} q_{1 j}^{\prime} D^{r_{j}} u_{j}+\sum_{j=1}^{p} \sum_{i=1}^{r_{j}-1} q_{i+1, j}^{\prime} D^{r_{j}-i} u_{j}+\sum_{j=1}^{p}\left(Q b_{r_{j}, i}+q_{r_{j}+1, j}^{\prime}-Q C_{A} Q^{-1} q_{r_{j}+1, j}^{\prime}\right) u_{j},
\end{aligned}
$$

where $b_{i j}^{T}=\left(b_{i j}, 0, \ldots, 0\right)$ being a $s$-real vector. Equation (A.6) can be rewritten as

$$
\dot{\bar{x}}=Q C_{A} Q^{-1} \bar{x}+Q\left(\sum_{j=1}^{p} b_{r_{j}, j}-C_{A} Q^{-1} q_{r_{j}, j}^{\prime}\right) u_{j}
$$

provided that $Q$ and $q(\cdot, \cdot)$ are chosen fulfilling

$$
q_{i j}^{\prime}=-Q b_{0 j} ; \quad q_{i+1, j}^{\prime}=-Q\left(b_{i j}-C_{A} Q^{-1} q_{i j}^{\prime}\right) ; \quad i=1,2, \ldots, r_{j}-1 ; j=1,2, \ldots, p .
$$

It is obvious that (A.7) and (A.8) is a well-posed state-space description of (A.1) with state vector $\bar{x}$ since the successive time-derivatives of the inputs are not forcing terms. Thus, any other state vector $z$ is generated as $z=T_{z} \bar{x}$ for some nonsingular $\left(\sum_{j=1}^{p} r_{j}+s\right)$ real constant matrix $T_{z}$. The proof of (i) has been completed. The proof of (ii) is similar by replacing the operator $D$ and the time-argument $(t)$ by their discrete counterparts $q$ and $[k]$, respectively.

Lemma A.2. The input-output description for system (2.1) at sampling instants is given by the difference equation

$$
A(q) y[k]=B(q) u[k]+\int_{0}^{T} \gamma^{T}(q, \tau) u(k T+\tau) d \tau
$$

for $k \geq n=n_{c}+n_{d}$, subject to initial conditions $y[j]=y_{j}$ for $j=0,1, \ldots, n-1$, where

$$
\begin{gathered}
A(q)=\operatorname{Det}(q I-\Phi) \sum_{i=0}^{n} a_{i} q^{n-i}, \quad B(q)=\gamma_{1}^{T}(q) \Gamma+d=\sum_{i=0}^{n} b_{i} q^{n-i}, \\
\gamma^{T}(q, \tau)=\gamma_{1}^{T}(q)\left(b_{c}^{T} e^{A_{c}^{T}(T-\tau)} \vdots 0^{T}\right)^{T}=\sum_{i=1}^{n} \gamma_{i}(\tau) q^{n-i} ; \\
\gamma_{1}^{T}(q)=c^{T} \operatorname{Adj}(q I-\Phi)=\sum_{i=1}^{n} \gamma_{i}^{1^{T}} q^{n-i} \quad \text { for } \tau \in[0, T), \\
\Phi=\left[\begin{array}{cc}
e^{A_{c} T} & e^{A_{c} T}\left(\int_{0}^{T} e^{-A_{c} \tau} d \tau\right) A_{d c} \\
A_{c d} & A_{d}
\end{array}\right] ; \quad \Gamma=\left\lfloor\begin{array}{c}
e^{A_{c} T}\left(\int_{0}^{T} e^{-A_{c} \tau} d \tau\right) b_{d c} \\
b_{d}
\end{array}\right], \\
c^{T}=\left(c_{c}^{T} \vdots c_{d}^{T}\right), \quad d=d_{c}+d_{d} .
\end{gathered}
$$


Proof. Direct calculations yield that the solution of (2.1) at sampling instants is described by the extended discrete system

$$
\begin{gathered}
x[k+1]=\Phi x[k]+\Gamma u[k]+\omega_{d}[k], \\
y[k]=c^{T} x[k]+d u[k]
\end{gathered}
$$

for all integer $k>0$ of state vector $x[k]=\left(x_{c}^{T}[k] \vdots x_{d}^{T}[k]\right)^{T}$ of dimension $n=n_{c}+n_{d}$, subject to the parameterizations (A.10), with $\omega_{d}[k]=\left(v_{d}[k], 0^{T}\right)^{T}=\left(\int_{0}^{T} b_{c}^{T} e^{A_{c}^{T}(T-\tau)} u(k T+\right.$ $\left.\tau) d \tau \vdots 0^{T}\right)^{T}$. The substitution of (A.11b) into (A.11a) yields directly

$$
y[k]=\frac{B(q)}{A(q)} u[k]+\frac{\gamma_{1}^{T}(q)}{A(q)} \omega_{d}[k]=\left(c^{T}(q I-\Phi)^{-1} \Gamma+d\right) u[k]+c^{T}(q I-\Phi)^{-1} \omega_{d}[k]
$$

for $k \geq n=n_{c}+n_{d}$ under initial conditions $y[j]=y_{j}$ for $j=0,1, \ldots, n-1$ since $\gamma_{1}^{T}(q) \omega_{d}[k]$ $=\int_{0}^{T} \gamma^{T}(q, \tau) u(k T+\tau) d \tau$. Equation (A.12) proves the result with $\operatorname{deg}(B) \leq \operatorname{deg}(A)=n$, with the degree inequality being strict if $d_{c}=-d_{d}$, and $\operatorname{Max}_{1 \leq i \leq n}\left(\operatorname{deg}\left(\gamma_{1 i}\right)\right)<\operatorname{deg}(A)$.

Proof of Lemma 2.1. The proof is addressed by analyzing the hybrid system (2.1) by evaluating the couplings from the digital sub-state to the continuous-time one by using Lemma A.2 which describes the nominal hybrid plant "at" sampling instants through a difference equation. Firstly, combining (2.1a) and (2.1c) with the formal use of $\dot{x}_{c}(t)=$ $D x_{c}(t)$ yields

$$
\alpha(D) y(t)=\beta(D) u(t)+\beta_{1}^{T}(D) \omega[k]+\alpha(D) z[k],
$$

where $\omega(\cdot)$ and $z(\cdot)$ are defined in $(2.3 \mathrm{c})$ for all time $t \in[k T,(k+1) T)$ and all integer $k \geq 0$. Taking Laplace transforms in (A.13), one gets

$$
y(s)=\frac{\beta(s)}{\alpha(s)} u(s)+\frac{i^{(k)}(s)}{\alpha(s)}+\frac{\beta_{1}^{T}(s) \omega(s)}{\alpha(s)}+z(s),
$$

where $i^{(k)}(s)=c_{c}^{T} \operatorname{Adj}\left(s I-A_{c}\right) x_{c}[k]$ with $x_{c}[k]=x_{c}(k T)$ is related to initial conditions of (A.13) on the interval $[k T,(k+1) T)$. The sampling interval initial conditions of the continuous-time filtered equations (2.3) are now introduced. Take Laplace transforms in (2.3) under (in general nonzero) initial conditions to yield

$$
\begin{array}{lr}
y(s)=F(s) y_{f}(s)-i_{y}(s) ; & u(s)=F(s) u_{f}(s)-i_{u}(s) \\
\omega(s)=F(s) \omega_{f}(s)-i_{\omega}(s) ; & z(s)=F(s) z_{f}(s)-i_{z}(s)
\end{array}
$$

with

$$
\begin{array}{ll}
i_{y}(s)=c_{F}^{T} \operatorname{Adj}\left(s I-A_{F}\right) x_{y}(0) ; & i_{u}(s)=c_{F}^{T} \operatorname{Adj}\left(s I-A_{F}\right) x_{u}(0) ; \\
i_{\omega}(s)=c_{F}^{T} \operatorname{Adj}\left(s I-A_{F}\right) x_{\omega}(0) ; & i_{z}(s)=c_{F}^{T} \operatorname{Adj}\left(s I-A_{F}\right) x_{z}(0),
\end{array}
$$


where $x_{y}(0), x_{u}(0), x_{\omega}(0)$ and $x_{z}(0)$ are initial conditions of the states of the realizations $R_{F}$ of the filtered equations (2.3a) and (2.3b). The substitution of (A.15) into (A.14) yields directly for the filtered variables

$$
\begin{aligned}
\alpha(s) y_{f}(s)= & \beta(s) u_{f}(s) \beta_{1}^{T}(s) \omega_{f}(s)+\alpha(s) z_{f}(s) \\
& +\left\{\frac{i^{(k)}(s)}{F(s)}+\frac{1}{F(s)}\left(\alpha(s)\left(i_{y}(s)-i_{z}(s)\right)-\beta(s) i_{u}(s)-\beta_{1}^{T}(s) i_{\omega}(s)\right)\right\}
\end{aligned}
$$

with the term $\{\cdot\}$ being associated with initial conditions of the various filters $(1 / F(s))$ used in (A.17). Equation (A.17) can be equivalently rewritten in the time-domain as

$$
\alpha(D) y_{f}(t)=\beta(D) u_{f}(t)+v(t)+v_{i c}(t)+v_{i c}(t)
$$

with

$$
\begin{aligned}
v(t) & =\beta_{1}^{T}(D) \omega_{f}(t)+\alpha(D) z_{f}(t) \\
& =\frac{1}{F(D)}\left(\beta_{1}^{T}(D)\left(A_{d c} x_{d}[k]+b_{d c} u[k]+\alpha(D)\left(c_{d}^{T} x_{d}[k]+d_{d} u[k]\right)\right)\right)
\end{aligned}
$$

for $t \in[k T,(k+1) T)$ subject to interval initial conditions where the signal $v_{i c}(t)$ is the Laplace inverse transform of $(1 / F(s))\left(\alpha(s)\left(i_{y}(s)-i_{z}(s)\right)-\beta(s) i_{u}(s)-\beta_{1}^{T}(s) i_{\omega}(s)\right)$ is defined in (2.8) as the response to initial conditions of the filters (2.3a) and (2.3b). To continue with the proof, note by applying Lemma A.1(ii) to the unfiltered $n$th order input-output description (A.9) and (A.10) "at" sampling instants driven by the sequences $\{u[k], k \geq 0\}$ and $\left\{\omega_{d}[k], k \geq 0\right\}$. Thus, it follows by construction that the state $x[k]$ is related by a real matrix transformation to the sequence of $3 n+2$ elements $\delta[k]$ defined by

$$
\begin{aligned}
\delta[k]= & \left(\delta_{y}^{T}[k] \vdots \delta_{u}^{T}[k] \vdots \delta_{\omega d}^{T}[k]\right)^{T} \\
= & (y[k], y[k-1], \ldots, y[k-n] \vdots u[k], u[k-1], \ldots, \\
& \left.u[k-n] \vdots \omega_{d}[k-1], \omega_{d}[k-2], \ldots, \omega_{d}[k-n]\right)^{T}
\end{aligned}
$$

for all $k \geq 0$ according to

$$
x_{d}[k]=E_{d} x[k]=E_{d} T_{d} \delta[k] ; \quad x_{c}[k]=E_{c} x[k]=E_{c} T_{d} \delta[k]
$$

since $\gamma_{1}^{T}(q) \omega_{d}[k]=\int_{0}^{T} \gamma^{T}(q, \tau) u(k T+\tau) d \tau$ where $E_{c}, E_{d}$, and $T_{d}$ are, respectively, $n_{c} \times n$, $n_{d} \times n$, and $n \times 3 n$ real matrices. From (A.9), (A.10), (A.20), and (A.21), one gets

$$
\begin{aligned}
v(t) & =\beta_{1}^{T}(D) \omega_{f}(t)+\alpha(D) z_{f}(t)=\frac{1}{F(D)}\left(\beta_{1}^{T}(D) Q_{d}+\alpha(D) q_{d}^{T}\right) \delta[k] \\
& =\sum_{i=0}^{n}\left(\frac{\beta_{1 i}(D)}{F(D)} y[k-i]+\frac{\beta_{2 i}(D)}{F(D)} y[k-i]+\sum_{i=1}^{n} \int_{0}^{T} \frac{\beta_{3 i}(D, \tau)}{F(D)} u[(k-1) T+\tau) d \tau\right]
\end{aligned}
$$


for $t \in[k T,(k+1) T]$ where

$$
Q_{d}=A_{d c} E_{d} T_{d}+b_{a} e_{n+1}^{T} ; \quad q_{d}^{T}=c_{d}^{T} E_{d} T_{d}+d_{d} e_{n+1}^{T}
$$

with $e_{i}$ being the $i$ th unity Euclidean vector and the coefficients of the polynomials $\beta_{i}^{T}(D)$ $=\left(\beta_{i 0}(D), \beta_{i 1}(D), \ldots, \beta_{i n}(D)\right)^{T}$ for $i=1,2$ and $\beta_{3 i}^{T}(D)=\left(b_{c}^{T} e^{A_{c}^{T}(T-\tau)} \beta_{3 i}^{\prime T}(D) \vdots 0^{T}\right)$ with $\beta_{3 i}^{\prime T}(D)$ $=\left(\beta_{3 i}^{\prime}(D), \beta_{32}^{\prime}(D), \ldots, \beta_{3 n}^{\prime}(D)\right)$ are fixed from the polynomial identity

$$
\left[\beta_{1}^{T}(D) \vdots \beta_{2}^{T}(D) \vdots \beta_{3}^{T}(D)\right]=c_{c}^{T} \operatorname{Adj}\left(D I-A_{c}\right) Q_{d}+q_{d}^{T}
$$

and the proof has been completed.

\section{B. Proofs related to stability results}

Proof of Lemma 4.1. (i) Consider the nonnegative function $V(t):=\int_{0}^{T} \tilde{\theta}^{T}(t, \tau) P^{-1}(t$, $\tau) \tilde{\theta}(t, \tau) d \tau$ for all $k \geq 0$. The following relationships hold:

$$
\begin{gathered}
\int_{0}^{T}\|\tilde{\theta}(t, \tau)\|^{2} d \tau \leq \sup _{0 \leq \tau \leq T}\left(\left[\lambda_{\max } P(t, \tau)\right]\right) V(t) \\
\int_{0}^{T}\left(\tilde{\theta}^{T}(t, \tau) \phi(t, \tau)\right)^{2} d \tau=\sup _{0 \leq t \leq \tau}\left(\|\phi(t, \tau)\|^{2}\right) \int_{0}^{T}\|\tilde{\theta}(t, \tau)\|^{2} d \tau \leq p(t) V(t) \\
b^{1 / 2}(t) e(e)\left(\eta_{f}(t)-e(t)\right) \leq b^{1 / 2}(t)\left(\zeta^{-1}-1\right) e^{2}(t) \leq 0
\end{gathered}
$$

since $\zeta>1$ and $b(t)=0$ if $|e(t)| \leq \zeta y(t)$. Direct calculations using (3.2), (3.3) and (B.1) lead to

$$
\begin{aligned}
& \dot{V}(t)= \int_{0}^{T}\left\{2 \dot{\tilde{\theta}}^{T}(t, \tau) P^{-1}(t, \tau) \tilde{\theta}(t, \tau)+\tilde{\theta}^{T}(t, \tau) \dot{P}^{-1}(t, \tau)\right\} d \tau \\
& \leq b(t)\left\{2 e(t)\left(\eta_{f}(t)-e(t)\right)\right. \\
&\left.\quad+b(t) \int_{0}^{T}\left[\tilde{\theta}^{T}(t, \tau)\left(\phi(t, \tau) \phi^{T}(t, \tau)-\lambda(t) P^{-1}(t, \tau)\right) \tilde{\theta}(t, \tau)\right] d \tau\right\} \\
& \leq-b(t)\left\{2\left(1-\zeta^{-1}\right) e^{2}(t)+(\lambda(t)-p(t)) V(t)\right\} \\
& \leq-\left(2 b(t)\left(1-\zeta^{-1}\right) e^{2}(t)+\lambda(t) V(t)\right) \leq 0 .
\end{aligned}
$$

Thus, $V(t) \leq V(0)<\infty$ is positive semidefinite and nonincreasing for all $t \geq 0$. Also, $\lambda(t) V(t)$ and $b(t) e^{2}(t)$ are bounded and converge asymptotically to zero. This implies that either $e(t)$ and/or $b(t)$ and either $V(t)$ and/or $\lambda(t)$ converge asymptotically to zero. If $e(t)$ converges asymptotically to zero then $b(t)$ also converges to zero from (3.3b). As a result, $b(t) \rightarrow 0$ as $t \rightarrow \infty$. Furthermore, $b(t) e^{2}(t) \rightarrow 0, b(t) V(t) \rightarrow 0$ and $b(t)(|e(t)|-$ $\left.\left|\eta_{f}(t)\right|\right)^{2} \leq b(t)\left(\eta_{f}(t)-e(t)\right)^{2} \rightarrow 0$ as $t \rightarrow \infty$. Also, $\lambda^{1 / 2} V^{1 / 2} \in L_{2} \cap L_{\infty}$ from (B.1c) and the fact that $b(t) e^{2}(t) \rightarrow 0$ as $t \rightarrow \infty$. Also, $b(t) \eta_{f}^{2}(t) \leq b(t) e^{2}(t)$ if $|e(t)| \geq \zeta \gamma(t) \geq\left|\eta_{f}(t)\right|$, and if $|e(t)|<\zeta \gamma(t)$ then $b(t)=0$. Thus, $b(t) \eta_{f}^{2} \rightarrow 0$ as $t \rightarrow \infty$. Also, $b(t) \gamma^{2} \leq b(t) e^{2}(t)$ if 
$|e(t)|<\zeta \gamma(t)$ (with $b(t)=0$ ) and if $e^{2}(t) \geq \zeta \gamma^{2}(t)>\gamma^{2}(t)$ as well. Thus, $b(t) \gamma^{2}(t) \rightarrow 0$ as $t \rightarrow \infty$. Thus, (i) has been fully proved.

(ii) Note that the covariance matrix is uniformly bounded by construction from (3.3b) and (3.3e). On the other hand, note from (3.3b) that

$$
\dot{P}^{-1}(t, \tau)=-P^{-1}(t, \tau) \dot{P}(t, \tau) P^{-1}(t, \tau)=-\lambda(t) P^{-1}(t, \tau)+b(t) \phi(t, \tau) \phi^{T}(t, \tau)
$$

which involves time-derivatives with respect to $t$. Note also that $b(t)\|\phi(t, \tau)\|^{2}$ is uniformly bounded and the forgetting factor is uniformly bounded and positively bounded from below for all time since $b(t) p(t)$ is bounded by construction from $(3.3 \mathrm{c})$ to $(3.3 \mathrm{~g})$. Thus, direct integration with respect to time $t$ from bounded initial conditions yields that the covariance inverse matrix is uniformly bounded with respect to time $t$ for all $\tau \in[0, T)$. Since the covariance matrix and its inverse are both uniformly bounded, what implies that they are both nonsingular for all time and that the integral matrix function that defines $V(t)$ is a continuous function on a finite interval, the parameter estimated vector is bounded for all time since $V(t)$ is bounded .

(iii) The property of square-integrability of the given signals follows directly from (B.9) and (B.2). The boundedness of the above signals remains still unproved. Now, note from (3.3b) that

$$
\begin{aligned}
\int_{0}^{t}\left\|\dot{\tilde{\theta}}\left(\tau^{\prime}, \tau\right)\right\| d \tau^{\prime} & \leq \int_{0}^{t}\left\|b\left(\tau^{\prime}, \tau\right) P\left(\tau^{\prime}, \tau\right)\right\| \phi\left(\tau^{\prime} \tau\right) e\left(\tau^{\prime}\right) \| d \tau^{\prime} \\
& \leq \sup _{0 \leq \tau^{\prime} \leq \tau}\left(\left\|b^{1 / 2}\left(\tau^{\prime}\right) P\left(\tau^{\prime}, \tau\right) \phi\left(\tau^{\prime}, \tau\right)\right\|\right) \int_{0}^{t} b^{1 / 2}\left(\tau^{\prime}\right)\left|e\left(\tau^{\prime}\right)\right| d \tau^{\prime}<\infty
\end{aligned}
$$

for all $t \geq 0$ and $\tau \in[0, T)$. Thus, $\dot{\hat{\theta}} \in L_{2}$ for all $\tau \in[0, T)$ (see $[6,8]$ ) and thus $\theta(t, \tau)$ converges for all $\tau \in[0, T)$ as $t \rightarrow \infty$. To prove, the uniform boundedness of this vector, note that (3.3a) can be rewritten as follows

$$
\dot{\hat{\theta}}(t, \tau)=b(t) P(t, \tau) \phi(t, \tau) \bar{\phi}(t) \frac{e(t)}{\bar{\phi}(t)} ; \quad \bar{\phi}(t):=\sup _{0 \leq \tau \leq T}(\|\phi(t, \tau)\|) .
$$

Since $b(t) P(t, \tau) \phi(t, \tau) \bar{\phi}(t)$ is bounded by construction, if $\dot{\hat{\theta}}(t, \tau)$ diverges then $|e(t)| / \bar{\phi}(t)$ diverges on the time interval $I_{a}:=\{t:|e(t)|>\zeta \gamma(t)\}$ since $\dot{\hat{\theta}}(t, \tau)$ is zero and then bounded for $t \notin I_{a}$. Since the parametrical error is uniformly upper-bounded from (ii) by a finite positive constant $k_{\theta}$, if $|e(t)| / \bar{\phi}(t)$ diverges then $\left|\eta_{f}(t)\right| / \bar{\phi}(t)$ diverges on $I_{a}$ from (3.2). Also, $k_{\theta} \bar{\phi}(t)+\left|\eta_{f}(t)\right|>\zeta \gamma(t) \Rightarrow \bar{\phi}(t)>\left((\zeta-1) / k_{\theta}\right) \gamma(t)$ for $t \notin I_{a}$ from (3.3f) and (3.3g) so that $\left(k_{\theta} /(\zeta-1)\right)\left(\left|\eta_{f}(t)\right| / \gamma(t)\right)>\left|\eta_{f}(t)\right| / \bar{\phi}(t) \rightarrow \infty$ on $I_{a}$ and $\left|\eta_{f}(t)\right| / \gamma(t)$ diverges on $I_{a}$. This contradicts Assumption 2.7 so that $\left|\eta_{f}(t)\right| / \bar{\phi}(t)$ and thus $|e(t)| / \bar{\phi}(t)$ and $\dot{\hat{\theta}}(t, \tau)$ cannot diverge on $I_{a}$ for any $\tau \in[0, T)$. Also, $b^{1 / 2}(t)|e(t)| \in L_{\infty}$ since $\dot{\hat{\theta}}(\cdot, \tau) \in L_{\infty}$ and $b^{1 / 2}(t) P(t) \phi(t) \in L_{\infty}$ for all $\tau \in[0, T)$. This fact leads directly to the remaining results of (iii). 
Proof of Theorem 4.2. The solution of (3.15) is

$$
\begin{aligned}
x(t)= & \psi_{A}\left(t, t_{0}\right) x(0) k^{*}[k] \frac{P(D, k T)}{A_{v}^{*}(D)}\left(A^{*}(D) y_{f}[k]-\hat{\beta}(D, k T) R(D, k T) y_{f}^{*}[k]\right. \\
& -L(D, k T) e[k]) \cdot \int_{0}^{t} \psi_{A}(t, \tau) \mu_{1} d \tau \\
& +\int_{0}^{t} \psi_{A}(t, \tau) \mu_{1}(\hat{\tilde{v}}(\tau)+e(\tau)) d \tau+x_{i c}(t)
\end{aligned}
$$

for all $t \in\left[t_{0}, \infty\right)$ and any $t_{0} \geq 0$ where $\psi_{A}(t, \tau)$ is the transition matrix associated with $A(t), x_{i c}(t)$ is a bounded signal associated with the contribution of the initial conditions of the filters together with the signal $r(t)=\hat{\beta}(D, t) R(D, t) y_{f}^{*}(t)$. The unforced system $x(t)=$ $\psi_{A}\left(t, t_{0}\right) x(0)$ is exponentially stable since (see $\left.[8,38]\right)$ :

(a) $A(t)$ is bounded and its eigenvalues are strictly inside the stability boundary for all $t$. Such a property follows from the boundedness of the estimates.

(b) $\int_{t_{1}}^{t_{1}+t}\|\dot{A}(\tau)\|^{2} \leq c_{1} t+c_{2}$ for all $t_{1}$ and some $t$ where $c_{1}$ is sufficiently small compared to the quotient of real constants $\sigma_{a} / \sigma_{r}$ where:

(1) $\left(-\sigma_{a}\right)<0$ is the stability abscissa of $\left[A(t)-(1 / 2) \Omega^{-1}(t) \dot{\Omega}(t)\right]$, where $\Omega(t)$ is the uniformly positive bounded definite solution to the Lyapunov's equation $A^{T}(t) \Omega(t)+\Omega(t) A(t)=-\Gamma$ with $\Gamma=\Gamma^{T}>0$.

(2) $\sigma_{r}>0$ is a real constant such that $\|\dot{\Omega}(t)\|_{2}^{2} \leq \sigma_{r}\|\dot{A}(t)\|_{2}^{2}$.

Note that $(b)$ follows from the fact that $\|\dot{\theta}\|^{2} \in L_{1} \cap L_{\infty}$. The diophantine equations (3.9) establish that for almost all $t$ (excluding perhaps sampling instants where the controller parameters have discontinuities) $(d / d t)(\hat{A} \cdot L+\hat{B} \cdot S)=0, \dot{L}$ and $\dot{R}$ exist at those instants since $\dot{\hat{A}}$ and $\dot{\hat{B}}$ exist. Each of the squares of the coefficients of the $\dot{L}$ and $\dot{R}$ polynomials is integrable and bounded on any finite time interval since for any coefficient $z(t)$ of the adaptive controller, $\int_{t_{1}}^{t+t_{1}} \dot{z}^{2}(\tau) d \tau=(1 / 3)\left(\dot{z}^{3}\left(t+t_{1}\right)-\dot{z}^{3}\left(t_{1}\right)\right)+K_{z}<\infty$ for any bounded function $\dot{z}$ on $\left(t_{1}, t+t_{1}\right)$ and $K_{z}$ is a bounded nonnegative real constant which over bounds the contributions of the all the eventual isolated discontinuities of $z$ at sampling instants within $\left[t_{1}, t+t_{1}\right]$ which make impulsive $\dot{z}$. As a result, $\|\dot{A}\|^{2}$ is integrable on any interval including a finite set of sampling instants. Now, it remains to guarantee that the unforced system is stable in order to prove (i). Direct calculations by using (3.1) and (3.7b) yield

$$
\begin{aligned}
|\hat{\tilde{v}}(t)| & =\left|\int_{0}^{T} \varphi^{T}(k T, \tau)(\hat{\theta}(t, \tau)-\hat{\theta}(k T, \tau)) d \tau+v_{0}(0, t)\right| \\
& \leq K_{\Delta \dot{\theta}}(t) \sup _{0 \leq \tau \leq T}\left(\|\varphi(k T, \tau)\|_{2}\right)+\left|v_{0}(0, t)\right|
\end{aligned}
$$

with $K_{\Delta \hat{\theta}}(t):=\int_{0}^{T}\left(\|\hat{\theta}(t, \tau)\|_{2}-\|\hat{\theta}(k T, \tau)\|_{2}\right) d t$ for all $t \in[k T,(k+1) T)$ and all integer $k \geq 0$ since $\hat{\tilde{v}}[k]=0$, and

$$
v_{0}(0, t)=c_{F}^{T} e^{A_{F} k T}\left(e^{A_{F}(t-k T)} \hat{\bar{x}}^{*}(0, t)-\hat{\bar{x}}^{*}(0, k T)\right)-e^{A_{F} k T}\left(\hat{\bar{x}}^{*}(0, t)-\hat{\bar{x}}^{*}(0, k T)\right)
$$


for all $t \in[k T,(k+1) T)$, all integer $k \geq 0$ is bounded and exponentially decaying. Note that

$$
|e(t)| \leq|e(t)-f(t)|+|f(t)| \leq g(t)+|f(t)| \leq \zeta\left(\varepsilon_{0}+\varepsilon \rho(t)\right)+|f(t)| .
$$

From (B.7) and (B.9), one gets directly:

$$
\begin{aligned}
&\left|\hat{\tilde{v}}(t)+e(t)+\frac{A^{*}(D)}{K[k] A_{v}^{*}(D)} y_{f}[k]-\frac{\hat{\beta}(D, k T) R(D, k T)}{K[k] A_{v}^{*}(D) F(D)} y^{*}[k]-\frac{L(D, k T)}{K[k] A_{v}^{*}(D)} e[k]\right| \\
& \leq k_{e}(0)+k_{r}(0)+\frac{\rho_{v}^{*}+1}{\rho_{v}^{*}}\left(\zeta \varepsilon_{0}+\varepsilon \sup _{0 \leq \tau \leq t}(\rho(\tau))+|f(t)|\right)+K_{\Delta \hat{\theta}}(t) \sup _{0 \leq \tau \leq t}\left(\|\varphi(k T, \tau)\|_{2}\right) \\
&+k_{1}(0)+\frac{1}{\rho_{v}^{*}} \cdot \sup _{0 \leq \tau \leq t}\left(\sup _{0 \leq \tau \leq t}\left(\|\varphi(t, \tau)\|_{2}\right)\right)+\left|v_{0}(0, t)\right|
\end{aligned}
$$

for all $t \in[k T,(k+1) T)$ and all integer $k \geq 0$, where:

(a) $k_{e}(0)$ being a finite nonnegative real constant associated with the response to the initial conditions a state-space realization of the strictly Hurwitz time-varying filters $L(D$, $k T) / K[k] A_{v}^{*}(D)$ and $\hat{\beta}(D, k T) R(D, k T) / K[k] A_{v}^{*}(D) F(D)$,

(b) $k_{r}(0)$ is a finite upper-bound on $[0, \infty)$ for the forced output sequence of $\hat{\beta}(D$, $k T) R(D, k T) / K[k] A_{v}^{*}(D) F(D)$ under the forcing sequence $\left\{y^{*}[k], k \geq 0\right\}$,

(c) $k_{1}(0)$ being a nonnegative real constant associated with the initial conditions of any state-space realization of the filter $1 / A_{v}^{*}(D)$ of stability abscissa $\left(-\rho_{v}^{*}\right)<0$.

Now, let $\left(-\rho^{*}\right)<0$ be stability abscissa of $A^{*}(D)$, that is, $\left\|\psi_{A}(t, \tau)\right\|_{2} \leq e^{-\rho^{*}(t-\tau)}$ for all $t$ and $\tau$. Note also that $\int_{t_{1}}^{t_{2}}\left\|\psi_{A}(t, \tau)\right\|_{2}|f(\tau)| d \tau \leq\left(1 / \sqrt{\rho^{*}}\right)\left(\int_{t_{1}}^{t_{2}} f^{2}(\tau) d \tau\right)^{1 / 2}$ by using the Cauchy-Schwartz inequality, which substituted, together with (B.10b) into (B.6) yields:

$$
\begin{aligned}
\sup _{t_{0} \leq \tau \leq t}\left(\|x(\tau)\|_{2}\right) \leq & \frac{1}{\rho^{*}}\left(\zeta \varepsilon \frac{\rho_{v}^{*}+1}{\rho_{v}^{*}}+\bar{K}_{\Delta \hat{\theta}}\left(t_{0}\right)+\frac{1}{\rho_{v}^{*}}\right) \cdot \sup _{t_{0} \leq \tau \leq t}\left(\|x(\tau)\|_{2}\right) \\
& +\frac{1}{\sqrt{\rho^{*}}}\left(\int_{t_{0}}^{t} f^{2}(\tau) d \tau\right)^{1 / 2}+k_{0}\left(t_{0}\right) \\
& +\frac{1}{\rho^{*}}\left(k_{e}\left(t_{0}\right)+k_{r}\left(t_{0}\right)+\left|v_{0}\left(t_{0}, t\right)\right|+\zeta \varepsilon_{0} \frac{\rho_{v}^{*}+1}{\rho_{v}^{*}}\right)
\end{aligned}
$$

since $\sup _{t_{0} \leq \tau \leq t}(\rho(t)) \leq \sup _{0 \leq \tau^{\prime} \leq t}\left(\sup _{t_{0} \leq \tau<\infty}\left(\left\|\varphi\left(\tau, \tau^{\prime}\right)\right\|_{2}\right)\right) \leq \sup _{t_{0} \leq \tau \leq t}\left(\|x(\tau)\|_{2}\right) \quad$ by construction (see (2.12), (2.14), and (3.16a)) where $\bar{K}_{\Delta \hat{\theta}}\left(t_{0}\right)=\sup _{t_{0} \leq \tau \leq \infty}\left(K_{\Delta \hat{\theta}}(\tau)\right)$. The real constants $k_{e}\left(t_{0}\right)$ and $k_{r}\left(t_{0}\right)$, which depend on $t_{0}$, and are defined on the interval $\left[t_{0}, \infty\right)$ similarly as $k_{e}(0)$ and $k_{r}(0)$ on $[0, \infty)$ and

$$
k_{0}\left(t_{0}\right)=\sup _{t_{0} \leq \tau \leq t}\left(\left\|x_{i c}(\tau)\right\|_{2}\right)+k_{1}\left(t_{0}\right)<\infty .
$$

Note also that $\bar{K}_{\Delta \hat{\theta}}\left(t_{0}\right)$ is bounded for any finite time $t_{0}>0$ and converges asymptotically to zero since the parameter estimates converge asymptotically from Lemma 4.1. 
Thus, there is a sufficiently large finite time $t_{0}$ such that $\|x(t)\|_{2}$ is bounded for $t \in\left[0, t_{0}\right)$ since from the structure of the solution (B.6), finite escape times cannot exist, and, furthermore, $\bar{K}_{\Delta \hat{\theta}}(t) \leq\left(\rho_{0}-\rho_{1}\right) / \rho_{v}^{*}$ for all $t \geq t_{0}$ where $\rho_{1} \in\left(0, \rho_{0}\right)$. It remains to be proved that $x(t)$ is bounded for all $t \geq t_{0}$. Now, define a normalized signal $\bar{f}(t)=f^{2}(t) /(1+$ $\left.\sup _{0 \leq \tau \leq T}\left(\|\varphi(t, \tau)\|_{2}\right)\right)^{1 / 2}$ from the signal $f(t)$ defined in $(3.3 \mathrm{~g})$ so that

$$
\frac{f^{2}(t)}{1+\sup _{0 \leq \tau \leq T}\left(\lambda_{\max }(P(t, \tau))\right) \sup _{0 \leq \tau \leq T}\left(\|\varphi(t, \tau)\|_{2}^{2}\right)} \leq k_{f} f^{2}(t)
$$

where $\infty>k_{f} \geq \sup _{0 \leq \tau \leq \infty}\left(\left(\bar{f}^{2}(t)+\sup _{0 \leq \tau \leq T}\left(\|\varphi(t, \tau)\|_{2}^{2}\right)\right) /\left(\bar{f}^{2}(t)+\sup _{0 \leq \tau \leq T}\left(\lambda_{\max }(P(t\right.\right.\right.$, $\left.\left.\tau))) \sup _{0 \leq \tau \leq T}\left(\|\varphi(t, \tau)\|_{2}^{2}\right)\right)\right)>0$. Thus, $\bar{f}^{2} \in L_{1}$ since $b(e-\bar{\eta})^{2} \in L_{1}$ from Lemma 4.1 so that $f^{2}(t) /\left(1+\sup _{0 \leq \tau \leq T}\left(\lambda_{\max }(P(t, \tau))\right) \sup _{0 \leq \tau \leq T}\left(\|\varphi(t, \tau)\|_{2}^{2}\right)\right)$ is integrable on $[0, \infty)$. This feature implies that $f^{2} \in L_{1}$ since $k_{f}$ is finite. Now, by construction of the regressor $x(\cdot)$, one has $k_{x} \sup _{t_{0} \leq \tau \leq t}\left(\|x(\tau)\|_{2}\right) \geq \sup _{t_{0} \leq \tau \leq t}\left(\sup _{0 \leq \tau \leq T}\left(\|\varphi(t, \tau)\|_{2}\right)\right)$ for some real constant $k_{x} \geq 1$ vector and the auxiliary state $x(t)$ (see (2.12a), (2.12b), (2.12c), (2.12d), (2.14), and (3.16a)). Thus, (B.13) into (B.12) leads to

$$
\sup _{t_{0} \leq \tau \leq t}\left(\|x(\tau)\|_{2}\right) \leq \frac{\rho_{v}^{*}}{\rho_{1}}\left(\omega\left(t_{0}\right)+\frac{1}{\sqrt{\rho^{*}}}\left(\int_{t_{0}}^{t} \bar{f}^{2}(\tau) d \tau+k_{x}^{2} \int_{t_{0}}^{t} \bar{f}^{2}(\tau) \sup _{t_{0} \leq \tau^{\prime} \leq \tau}\left(\left\|x\left(\tau^{\prime}\right)\right\|_{2}^{2}\right) d \tau\right)^{1 / 2}\right),
$$

where $\omega\left(t_{0}\right)=k_{0}\left(t_{0}\right)+\left(1 / \rho^{*}\right)\left(k_{e}\left(t_{0}\right)+k_{r}\left(t_{0}\right)+\left|v_{0}\left(t_{0}, t\right)\right|+\zeta \varepsilon_{0}\left(\left(\rho_{v}^{*}+1\right) / \rho_{v}^{*}\right)\right)$ after using the constraint: $0<1 /\left(\rho^{*}-\left(\zeta \varepsilon\left(\rho_{v}^{*}+1\right) / \rho_{v}^{*}\right)-\left(\left(1+\rho_{0}-\rho_{1}\right) / \rho_{v}^{*}\right)\right) \leq \rho_{v}^{*} / \rho_{1}$ that holds since $\varepsilon<\left(\rho^{*} \rho_{v}^{*}-1-\rho_{0}\right) / 2 \zeta\left(\rho_{v}^{*}+1\right)$. Now, it follows by taking squares in both sides of (B.14) that

$$
\begin{aligned}
& \sup _{t_{0} \leq \tau \leq t}\left(\|x(\tau)\|_{2}^{2}\right) \\
& \quad \leq 2\left(\frac{\rho_{v}^{*}}{\rho_{1}}\right)^{2}\left(\omega^{2}\left(t_{0}\right)+\frac{1}{\rho^{*}}\left(\int_{t_{0}}^{t} \bar{f}^{2}(\tau) d \tau+k_{x}^{2} \int_{t_{0}}^{t} \bar{f}^{2}(\tau) \sup _{t_{0} \leq \tau^{\prime} \leq \tau}\left(\left\|x\left(\tau^{\prime}\right)\right\|_{2}^{2}\right) d \tau\right)\right) .
\end{aligned}
$$

Thus, since $\bar{f}^{2} \in L_{1}$ and $\omega\left(t_{0}\right)$ is bounded for all finite $t_{0}$, it follows from the application of Gronwall's lemma to (B.15) that $x(t)$ is also bounded for all $t \geq t_{0}$ since $\|x(t)\|_{2}^{2} \leq$ $k_{3}\left(t_{0}, t\right) e^{k_{4}\left(t_{0}, t\right)}$ for all $t \geq t_{0}$, where

$$
\begin{gathered}
k_{3}\left(t_{0}, t\right)=2\left(\frac{\rho_{v}^{*}}{\rho_{1}}\right)^{2}\left(\omega^{2}\left(t_{0}\right)+\frac{1}{\rho^{*}} \int_{t_{0}}^{t} \bar{f}^{2}(\tau) d \tau\right) \leq k_{3}<\infty, \\
k_{4}\left(t_{0}, t\right)=\frac{2}{\rho^{*}}\left(\frac{\rho_{v}^{*} k_{x}}{\rho_{1}}\right)^{2} \int_{t_{0}}^{t} \bar{f}^{2}(\tau) d \tau \leq k_{4}<\infty
\end{gathered}
$$

This implies that $\dot{x}(t)$ is also bounded from (B.16). As a result, $D^{j} y_{f}(t)$ and $D^{i} u_{f}(t)$; $i=0,1, \ldots, n_{c}$ are bounded for all $t \geq 0$. The unfiltered output and input signals are also bounded for all time what follows $u(t)=F(D) u_{f}(t)$ and $y(t)=F(D) y_{f}(t)$. The proof of (i) has been completed. To prove (ii), note that $x_{i c}(t)$ converges asymptotically to zero 
and rewrite $(3.13)$ as

$$
\begin{aligned}
\hat{\alpha}(D, t)\left(y_{f}(t)-y_{f}^{*}(t)\right)= & \hat{\beta}(D, t) u_{f}(t)+\frac{1}{K^{*}[k] A_{v}^{*}(D)} A^{*}(D)\left(y_{f}(t)-y_{f}^{*}(t)\right) \\
& +\left(\frac{\hat{\alpha}(D, t) L(D, t)}{K^{*}[k] A_{v}^{*}(D)}-\hat{\alpha}(D, t)\right) y_{f}^{*}(t)-L(D, k T) e[k]+\hat{\tilde{v}}(t)+e(t) .
\end{aligned}
$$

Equation (B.17), together with (3.8), allows reconstructing the auxiliary system (3.15) accordingly to obtain again (B.16), subject to (B.17), where now $k_{r}\left(t_{0}\right)$ is an absolute upperbound of the forced response of the exponentially stable filter $\left(\left(L(D, t) / A_{v}^{*}(D)\right)-1\right)(\hat{\alpha}(D$, $t) / F(D))$ on $\left[t_{0}, \infty\right)$ when driven by $y^{*}(t)$ where the finite time instant $t_{0}$ is chosen as in (i). From (i), all the filtered signals in the loop are bounded for all time. Also, $\hat{\tilde{v}}(t) \rightarrow 0$ as $t \rightarrow 0$ from $(3.7 \mathrm{c})$ since $(1 / F(D))$ is a exponentially stable filter, so that $v_{0}(t)$ is exponentially decaying, and all the parameter estimates converge to a finite limit. For some sufficiently large time $t_{0}^{\prime} \geq t_{0}, k_{r}\left(t_{0}^{\prime}\right) \leq\left(k_{r} / \rho^{*} \rho_{v}^{*}\right) \sup _{t_{0}^{\prime} \leq \tau<\infty}\left(\left|y^{*}(\tau)\right|\right)$ is arbitrarily small for some constant $K_{r}<1$ since the reference output is arbitrarily small for $t \geq t_{0}^{\prime}$. Thus, since $k_{e}(t)$ and $k_{0}(t)$ converge asymptotically zero as time tends to infinity, $\omega\left(t_{0}^{\prime}\right) \leq\left(K_{\omega} / \rho^{*} \rho_{v}^{*}\right)$ $\sup _{t_{0}^{\prime} \leq \tau<\infty}\left(\left|y^{*}(\tau)\right|\right)+K_{\omega}\left(t_{0}^{\prime}\right)$ for $\varepsilon_{0}=0$ with some constant $K_{\omega}<1$ and $K_{\omega}\left(t_{0}^{\prime}\right)$ being arbitrarily small. Thus, $K_{3}\left(t_{0}^{\prime}, t\right)$ converges to zero as $t_{0}^{\prime}$ becomes arbitrarily large. As a result, the state of the auxiliary system built with (3.8) converges asymptotically to zero as time tends to infinity and proposition (ii) follows directly.

\section{References}

[1] A. Bilbao-Guillerna, M. De la Sen, A. Ibeas, and S. Alonso-Quesada, Robustly stable multiestimation scheme for adaptive control and identification with model reduction issues, Discrete Dyn. Nat. Soc. 2005 (2005), no. 1, 31-67.

[2] P. A. Bonatti and A. Peron, On the undecidability of logics with converse, nominals, recursion and counting, Artificial Intelligence 158 (2004), no. 1, 75-96.

[3] R. Cristi and R. V. Monopoli, Model reference adaptive control: the hybrid approach, Proc. Automat. Contr. Conf., 1982, pp. 848-851.

[4] A. Datta and J. Ochoa, Adaptive internal model control: design and stability analysis, Automatica J. IFAC 32 (1996), no. 2, 261-266.

[5] M. De la Sen, Adaptive sampling for improving the adaptation transients in hybrid adaptive control, Internat. J. Control 41 (1985), no. 5, 1189-1205.

[6] Multirate hybrid adaptive control, IEEE Trans. Automat. Control 31 (1986), no. 6, 582-586.

[7] - The reachability and observability of hybrid multirate sampling linear systems, Comput. Math. Appl. 31 (1996), no. 1, 109-122.

[8] Robust adaptive stabilization of time-invariant first-order hybrid systems with covariance resetting, Internat. J. Non-Linear Mech. 33 (1998), no. 1, 47-57.

[9] M. De la Sen and J. Jugo, Robust direct adaptive control for a class of systems with delays, IMA J. Math. Control Inform. 15 (1998), no. 1, 25-52.

[10] M. De la Sen and N. Luo, On the uniform exponential stability of a wide class of linear time-delay systems, J. Math. Anal. Appl. 289 (2004), no. 2, 456-476.

[11] M. De la Sen and I. Sagastabeitia, Compensation of uncertain continuous systems by using the internal model control principle, Internat. J. Systems Sci. 26 (1995), no. 5, 1153-1180. 
[12] Ph. de Larminat, On the stabilizability condition in indirect adaptive control, Automatica J. IFAC 20 (1984), no. 6, 793-795.

[13] C. A. Desoer and M. Vidyasagar, Feedback Systems: Input-Output Properties, Academic Press, New York, 1995.

[14] G. Feng, A new algorithm for continuous-time robust adaptive control, Proceedings of the 34th Conference on Decision and Control, vol. 1, IEEE- Institute of Electrical and Electronics Engineers, Louisiana, 1995, pp. 165-169.

[15] G. Ferrari-Trecate, E. Gallestey, P. Letizia, M. Spedicato, M. Morari, and M. Antoine, Modeling and control of co-generation power plants: a hybrid system approach, IEEE Trans. Contr. Syst. Technol. 12 (2004), no. 5, 694-705.

[16] P. J. Gawthrop, Hybrid self-tuning control, Proc. IEE-D 127 (1980), no. 5, 229-236.

[17] F. Giri, J. M. Dion, M. M'Saad, and L. Dugard, A globally convergent pole placement indirect adaptive controller, IEEE Trans. Automat. Control 34 (1989), no. 3, 353-356.

[18] W. M. Haddad and V. Chellaboina, Dissipativity theory and stability of feedback interconnections for hybrid dynamical systems, Math. Probl. Eng. 7 (2001), no. 4, 299-335.

[19] W. M. Haddad, V. Chellaboina, and S. G. Nersesov, Hybrid nonnegative and compartmental dynamical systems, Math. Probl. Eng. 8 (2002), no. 6, 493-515.

[20] K. Hörnell and P. Lötstedt, Adaptive iteration to steady state of flow problems, J. Sci. Comput. 20 (2004), no. 3, 331-354.

[21] P. I. Ioannou and A. Datta, Robust adaptive control: a unified approach, Proc. IEEE 79 (1991), no. $12,1736-1768$.

[22] P. Jegou and C. Terrioux, Bactracking bounded tree-decomposition of constraint networks, Artificial Intelligence 146 (2003), no. 1, 75-96.

[23] D. Kincaid and W. Cheney, Numerical Analysis, Brooks/Cole Publishing, California, 1991.

[24] B. Kuo, Automatic Control Systems, Prentice-Hall, New Jersey, 1968.

[25] C.-H. Lin, W.-D. Chou, and F.-J. Lin, Adaptive hybrid control using a recurrent neural network for a linear synchronous motor servo-drive system, IEE P-Contr. Theor. Ap. 148 (2001), no. 2, $156-168$.

[26] Z. Liu, C. Li, and W. Xu, Hybrid control of biped robots in the double-support phase via H-infinity approach and fuzzy neural networks, IEE P-Contr. Theor. Ap. 150 (2003), no. 4, 347-354.

[27] H. R. Marzban and M. Razzaghi, Optimal control of linear delay systems via hybrid of block-pulse and Legendre polynomials, J. Franklin Inst. 341 (2004), no. 3, 279-293.

[28] I. M. Mitchell and C. J. Tomlin, Overapproximating reachable sets by Hamilton-Jacobi projections, J. Sci. Comput. 19 (2003), no. 1-3, 323-346.

[29] K. S. Narendra, I. H. Khalifa, and A. M. Annaswamy, Error models for stable hybrid adaptive systems, IEEE Trans. Automat. Control 30 (1985), no. 4, 339-347.

[30] Y. Niu, J. Lam, and X. Wang, Sliding mode control for uncertain neutral delay systems, IEE PContr. Theor. Ap. 151 (2004), no. 1, 38-44.

[31] Y. Niu, J. Lam, X. Wang, and D. W. C. Ho, Observer-based sliding mode control for nonlinear state-delayed systems, Internat. J. Systems Sci. 35 (2004), no. 2, 139-150.

[32] K. A. Ossman and E. W. Kamen, Adaptive regulation of MIMO linear discrete-time systems without requiring a persistent excitation, IEEE Trans. Automat. Control 32 (1987), no. 5, 397404.

[33] C. Samson and J.-J. Fuchs, Discrete adaptive regulation of not-necessarily minimum-phase systems, Proc. IEE-D 128 (1981), no. 3, 102-108.

[34] M. Sintzoff, Iterative synthesis of control guards ensuring invariance and inevitability in discretedecision games, From Object-Orientation to Formal Methods, Lecture Notes in Computer Science, vol. 2635, 2004, pp. 272-301.

[35] B. Sjögreen and H. C. Yee, Multiresolution wavelet based adaptive numerical dissipation control for high order methods, J. Sci. Comput. 20 (2004), no. 2, 211-255. 
[36] F. Valenciaga, P. F. Puleston, and P. E. Battaiotto, Variable structure system control design method based on a differential geometric approach: application to a wind energy conversion subsystem, IEE P-Contr. Theor. Ap. 151 (2004), no. 1, 6-12.

[37] R.-J. Wai, C.-M. Lin, and C.-F. Hsu, Hybrid control for induction servomotor drive, IEE P-Contr. Theor. Ap. 149 (2002), no. 6, 555-562.

[38] Q. H. Wu, Y. Liu, and Y. H. Zhang, Adaptive control for induction servo motor based on wavelet neural networks, Advances in Neural Networks, International Symposium on Neural Networks 2004, Part 2, Lecture Notes in Computer Science, no. 3174, Springer, Berlin, 2004, pp. 156-162.

[39] L. Xo and W. Yu, Robust adaptive control using neural networks and projection, Advances in Neural Networks, International Symposium on Neural Networks 2004, Part 2, Lecture Notes in Computer Science, no. 3174, Springer, Berlin, 2004, pp. 77-82.

[40] L. A. Zadeh and C. A. Desoer, Linear Systems Theory. The State Space Approach, McGraw-Hill, New York, 1963.

M. de la Sen: Department of Electricity and Electronics, Faculty of Science and Technology, University of Basque Country, Leioa (Bizkaia), Aptdo, 644 de Bilbao, Spain

E-mail address: wepdepam@lg.ehu.es 


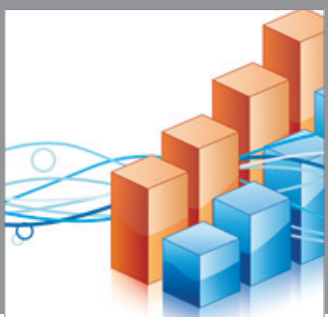

Advances in

Operations Research

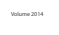

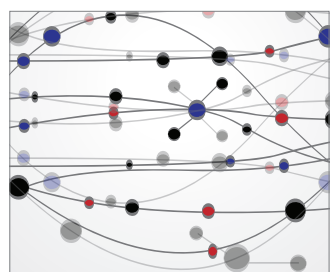

\section{The Scientific} World Journal
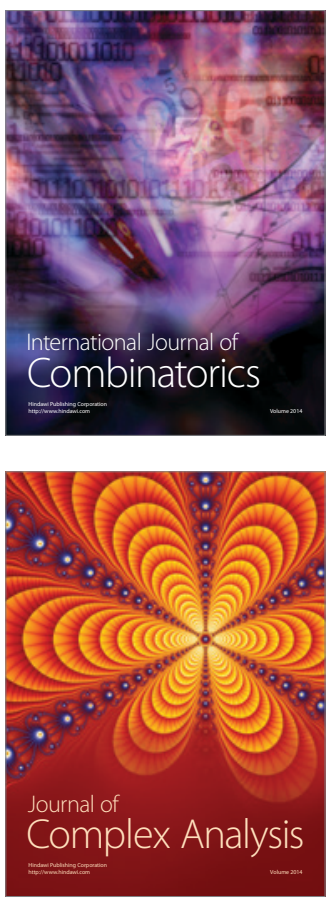

International Journal of

Mathematics and

Mathematical

Sciences
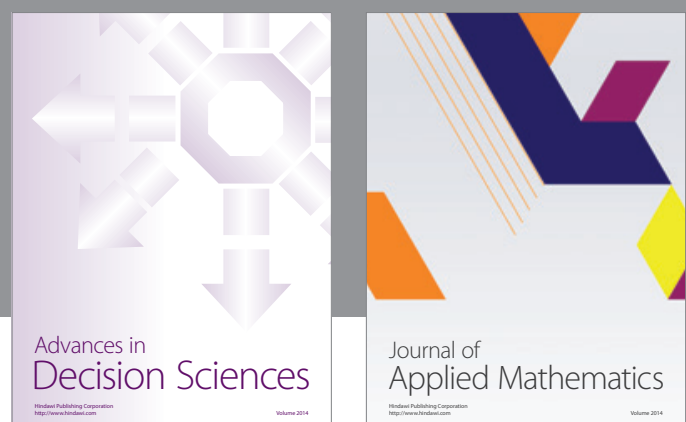

Journal of

Applied Mathematics
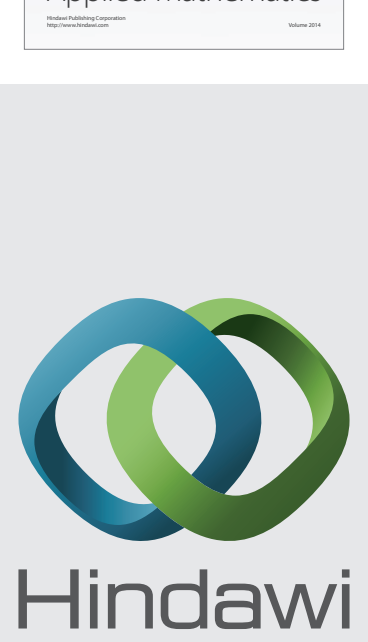

Submit your manuscripts at http://www.hindawi.com
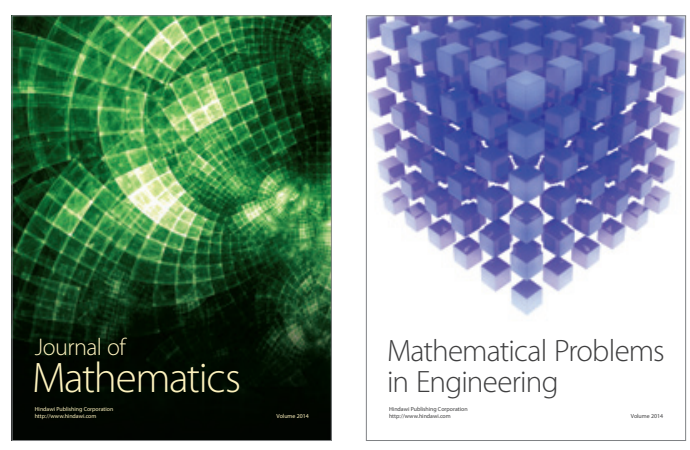

Mathematical Problems in Engineering
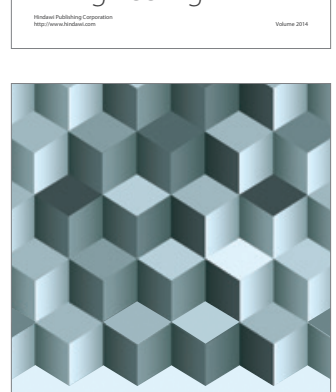

Journal of

Function Spaces
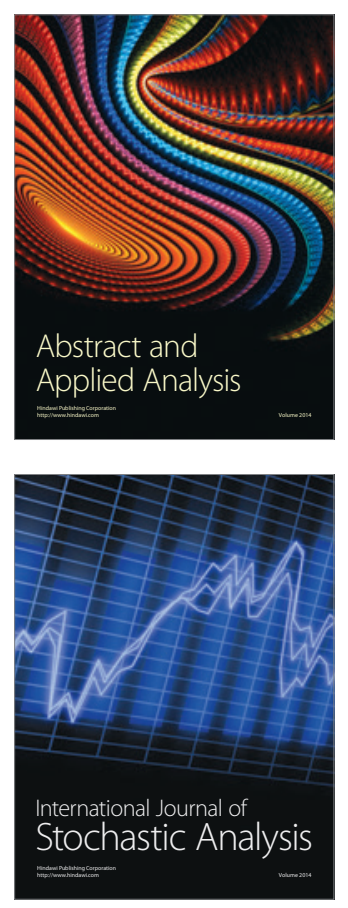

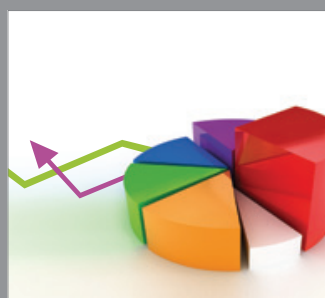

ournal of

Probability and Statistics

Promensencen
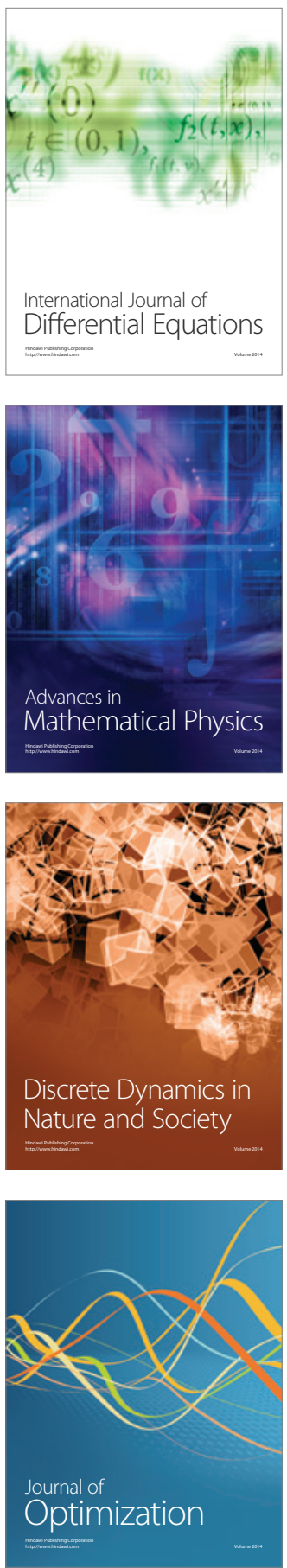